\title{
Analysis of Systematic Errors in Geocenter Coordinates Determined From GNSS, SLR, DORIS, and GRACE
}

\author{
Wiesław Kosek, ${ }^{1}$ (D) Waldemar Popiński, ${ }^{4}$ Agnieszka Wnenk, ${ }^{2}$ (i) Krzysztof Sośnica, ${ }^{3}$ \\ and Maria ZBYLUT-Górska ${ }^{2}$
}

\begin{abstract}
The goal of this paper is to determine and analyze the common geocenter signal from the geocenter coordinates based on four independent techniques: Doppler Orbitography and Radiopositioning Integrated by Satellite (DORIS), Global Navigation Satellite System (GNSS), Gravity Recovery And Climate Experiment with the ocean bottom pressure model, and Satellite Laser Ranging, and to analyze the residuals as the differences between these geocenter coordinates and their common signal. Another objective of this paper is to compute variable amplitudes and phases of the annual and semi-annual oscillations in the geocenter coordinates of these techniques by the combination of the Fourier Transform Band Pass Filter (FTBPF) with the Hilbert Transform (FTBPF + HT) and to compare their mean values with those obtained by other authors. It was assumed that the geocenter time series of individual techniques consist of the common signal of geocenter motion, systematic errors resulting from orbital modeling and noise. Generally, the annual oscillation amplitudes in these techniques computed by the FTBPF + HT vary in time and their mean values are of the order of $2 \mathrm{~mm}$ for the $X$ coordinate, 2.4-3.6 $\mathrm{mm}$ for the $\mathrm{Y}$ coordinate and $2.8-5.6 \mathrm{~mm}$ for the $\mathrm{Z}$ coordinate and the semi-annual oscillation amplitude is variable and about two times smaller than the annual one. The phases of these two oscillations are also variable, there are differences in their mean values for different techniques and the semi-annual oscillation phases changes throughout the entire phase range. To detect the common geocenter signal the wavelet-based semblance filtering (WBSF) method was applied. The weighted mean model was computed from all geocenter coordinate pairs from individual techniques assuming weights as inversely proportional to the variances of differences between the geocenter coordinates and their corresponding WBSF outputs. The average and median models computed from these outputs show a good agreement with the weighted mean model and generally, the average amplitudes of the annual signal in these models are of the order of $2 \mathrm{~mm}$ in each
\end{abstract}

1 Faculty of Civil Engineering and Geodesy, Military University of Technology, Warsaw, Poland. E-mail: wieslaw.kosek@wat.edu.pl

2 Department of Land Surveying, Faculty of Environmental Engineering and Land Surveying, University of Agriculture in Krakow, Krakow, Poland.

3 Institute of Geodesy and Geoinformatics, Wroclaw University of Environmental and Life Sciences, Wroclaw, Poland.

4 Statistics Poland, Warsaw, Poland. geocenter coordinate. The FTBPF amplitude spectra of these models reveals the retrograde annual oscillation in the XY equatorial plane. The FTBPF and FTBPF + HT amplitude spectra of geocenter time series and their residuals show mainly the maxima of different heights in the annual frequency band. The annual oscillations left in all residuals and oscillations with period less than $\sim 120$ days in DORIS and GNSS amplitude spectra may be caused by systematic errors of techniques resulting from mismodeling of satellite orbits.

Keywords: geocenter, wavelet semblance filtering, DORIS, GNSS, SLR, GRACE.

\section{Introduction}

\subsection{Definition of the geocenter motion}

Geocenter variation is caused by mass redistribution in the Earth's fluid layers. It is usually defined as the motion of the center of mass (CM) of the whole Earth including atmosphere, oceans and continental water with respect to the center of the solid Earth which is recognized as the center of figure $(\mathrm{CF})$ (e.g. Feissel-Vernier et al. 2006; Klemann and Martinec 2009; Wu et al. 2012). In some studies, the geocenter motion is considered as the motion of the CF with respect to the CM (e.g. Dong et al. 1997, 2003). The International Earth Rotation and Reference Systems Service (IERS) Conventions 2010 define the geocenter motion as the instantaneous $\mathrm{CM}$ variations with respect to the origin of the International Terrestrial Reference Frame (ITRF) (Petit and Luzum 2010). However, some applications require a reference to the instantaneous CM. Therefore, it is recommended to use the annual geocenter motion model derived from the same SLR series used to define the ITRF2014 long term origin (Altamimi et al. 2016). Furthermore, 
geocenter motion can be determined on the basis of observations from space geodetic networks which consist of a finite number of globally but not homogeneously distributed stations. Therefore, a network of tracking stations realizes Center of Network $(\mathrm{CN})$ and the discrepancy between $\mathrm{CN}$ and $\mathrm{CF}$ is known as the network effect (Wu et al. 2002).

The recent development of the satellite geodetic techniques enables observing and measuring geodynamic processes caused by mass redistribution in the Earth's fluid layers, such as the geocenter motion, plate tectonic motion, as well as irregularities in Earth rotation and sea level variations. Therefore, one of the fundamental tasks of modern geodesy is the maintenance and continuous development of the ITRF with the associated precise determination of the position of the instantaneous CM in the adopted reference frame at a given time. The position of the instantaneous $\mathrm{CM}$ has a significant impact on the measurement accuracy of geodynamic processes (Kuang et al. 2015; Wu et al. 2011, 2012). It is due to the differences between the CM and the ITRF origin, which may cause systematic errors in satellite orbits and stations positions. The reason is that satellites orbit around the instantaneous CM, whereas their orbits and station coordinates are referred to the ITRF. Neglecting geocenter motion may have a crucial impact on the recovery of mass variability derived from satellite gravimetry and large-scale inter-basin ocean mass exchange (Chen et al. 2005). Chambers et al. (2004) found differences in annual amplitudes of the order of $15 \%$ of the mean sea level variations between Gravity Recovery And Climate Experiment (GRACE) and Jason-1 altimeter data when neglecting the contribution of the geocenter motion. These differences could be reduced to the level of $1 \%$ when considering the seasonal variations of the geocenter motion.

The ITRF2014, as one of the realizations of the International Terrestrial Reference System (ITRS), is derived from observations of four space-geodetic techniques (Altamimi et al. 2016). The ITRF origin is defined as the mean $\mathrm{CM}$ averaged over long time span of the satellite laser ranging (SLR) observations. In the case of the ITRF2014 it is specified that there are zero translation parameters at epoch 2010.0 and zero translation rates with respect to the international laser ranging service (ILRS) SLR time series (Altamimi et al. 2016). According to Altamimi et al. (2016), the ITRF2014 is generated with improved modeling of nonlinear station motions, including annual and semi-annual signals of station positions and postseismic deformation for sites that were subject to major earthquakes. However, the origin of the ITRF2014 follows the average CM linearly with time, thus for seasonal and short time scales the ITRF origin is closer to the CF rather than the CM. For secular time scales the ITRF origin is at the CM (Collilieux et al. 2009; Dong et al. 2003; Wu et al. 2012).

Another realization of ITRS is the JTRF2014 developed by Jet Propulsion Laboratory (JPL) based upon the Kalman filter and smoother algorithms (Wu et al. 2015, Abbondanza et al. 2017). This is a subsecular, time-series based terrestrial reference frame whose origin is at the quasi-instantaneous $\mathrm{CM}$ as detected by the SLR technique.

\subsection{Recovery of the Geocenter Motion}

In general, observational techniques of space geodesy can determine geocenter motion based on four major methods: (1) translational approach, (2) direct approach (3) inverse approach, and (4) hybrid approach.

The first approach results from the fact that satellites orbit around the CM and are tracked by stations located on the solid Earth surface. This approach seeks to determine mean geocenter motion time series from a network of satellite tracking ground stations (Wu et al. 2017). Thus, CM motions with respect to $\mathrm{CN}$ are determined and this method is often called "network shift approach". In the second approach the geocenter motion is estimated from multi-satellite data. The displacements with acceleration parameters are estimated for entire network of satellite tracking stations. Subsequently, estimated stations coordinates are then projected into ITRF using Helmert transformation which parameters represent the origin difference between the estimated displaced stations network and ITRF (Cheng et al. 2013). This approach is used for the SLR technique where geocenter motion is usually not modelled in precise orbit determination (Cheng et al. 2013). In the 
third method of the geocenter recovery, it is assumed that mass redistributions within the solid Earth and its fluid layers cause changes of the first degree Stokes coefficients of the gravitational potential. In the reference frame with the origin in the $\mathrm{CM}$ these coefficients are equal to zero, whereas in the reference frame associated with the Earth's surface, such as ITRF2014, degree one coefficients are different from zero and the vector of geocenter position represents the distance between the $\mathrm{CM}$ and the $\mathrm{CF}$ (Bouille et al. 2000; Dong et al. 2003; Guo et al. 2008; Kang et al. 2009). The first degree Stokes coefficients of the gravitational potential can be recovered using the method initially proposed by Swenson et al. (2008) which is based on GRACE data and the ocean bottom pressure (OBP) model. The time series of degree one coefficients $\left(\mathrm{C}_{10}, \mathrm{C}_{11}, \mathrm{~S}_{11}\right)$ can subsequently be used to infer geocenter motion. In the inverse approaches the deformations of the Earth's crust due to mass redistributions (Blewitt et al. 2001) could also be used to recover geocenter motion. These deformations can be observed by globally distributed geodetic tracking network of global positioning system (GPS) stations (Blewitt and Clarke 2003; Rietbroek et al. 2012) or SLR and Global Navigation Satellite System (GNSS) stations (Glaser et al. 2015a, b). In the fourth approach, geocenter motion is recovered using the translational approach as well as surface deformations related with mass redistributions from the inverse approaches (Blewitt et al. 2001; Lavallée et al. 2006; Collilieux et al. 2012; Wu et al. 2017).

\subsection{Motivation and Goals of This Paper}

The purpose of this paper is to determine the stochastic geocenter motion model using the waveletbased semblance filtering (WBSF) (Kosek et al. 2014) of the SLR, GNSS, GRACE + OBP, Doppler Orbitography and Radiopositioning Integrated by Satellite (DORIS) geocenter coordinates by extracting the common signals detected in pairs of time series of all techniques.

According to Glasser et al. (2015a, b), determination of geocenter motion by combination of different techniques is more robust and less affected by technique-specific issues, such as orbit modeling deficiencies, limited observation sensitivity to some of the geocenter components or limited and irregular spatial distribution of stations, than the individual solutions. SLR is thought to be the most robust technique to extract the geocenter signals. However, the limited number of SLR stations in some areas, especially on the southern hemisphere, reduces the sensitivity of this technique to crustal displacements caused by surface mass redistributions. For example, the impact of the crust deformations caused by the atmospheric pressure loading on the geocenter motion cannot be fully reconstructed by SLR results due to the inhomogeneity of the station distribution (Sośnica et al. 2013). DORIS and GNSS stations have a much better global distribution. However, the GNSS-derived geocenter motion is subject to modeling deficiencies and systematic errors in clock and troposphere estimates, antenna offsets and orbit modeling issues (Meindl et al. 2013; Rebischung et al. 2013) especially for the $Z$ component. The GNSS-derived geocenter variations may indicate some offsets, but the phases of the dominating annual oscillation typically agree with the SLR results for the equatorial $\mathrm{X}$ and $\mathrm{Y}$ components (Rebischung et al. 2016a, b; Thaller et al. 2014). Moreover, new solutions are also being introduced to improve the quality of geocenter motion determination from GPS observations. For example Haines et al. (2015) added GRACE GPS tracking data to the GPS ground tracking network and significantly improved the repeatability of the geocenter determination.

The proper geocenter model is relevant for other geodetic observation techniques that require instantaneous CM positions e.g. satellite altimetry or satellite gravimetry (Chambers et al. 2004). Two time series can be compared by calculating the correlation coefficient or coherence between them in time or in time-frequency domains, respectively. The wavelet analysis enables the location of common signals in the time and frequency domains (Cheng and Tapley 2004) and can successfully be applied to the extraction of signals with varying amplitudes and phases. Based on the known literature on time series analysis, the wavelet-based semblance filtering (WBSF) seems to be the most appropriate method to calculate the common signals in all frequency bands in two time series. In this paper, we search for 
the common signals which comprise broadband oscillations in all frequency bands.

The GNSS, SLR, as well as DORIS geocenter time series are determined using the translational approach, whereas the GRACE + OBP series is computed based on the inverse approach. Consequently, the GRACE + OBP geocenter time series are included in this analysis as a series determined by an independent method providing additional information on the geocenter motion phenomenon in relation to the other time series. Finally, this paper examines the consistency of the mean amplitudes and phases of the annual and semi-annual oscillations and time variability of the weighted, average and median geocenter time series models derived using independent space geodetic techniques.

\section{Input Data}

Mass redistribution within the atmosphere, ocean and continental hydrology influence the geocenter motion. The impact of the atmosphere on geocenter motion can be specified using surface pressure data, which in turn can be obtained on the basis of data provided by climate and weather organizations. In the case of oceanic response to the surface pressure variations, the information from the modified inverted barometer and non-inverted barometer models can be used. The effect of ocean mass redistributions on geocenter variations can be obtained using satellite altimetry observations including the effects of steric sea level variations, or solutions of oceanic general circulation models. Hydrological mass redistributions in the continents include snow, soil moisture, ice sheet, glacier as well as surface water and ground water. Information about these mass redistributions can be obtained from land surface models. More information about the impact of the mass loading on the geocenter motions can be found in e.g. Dong et al. (2014).

\subsection{SLR Solution}

In this study, we use monthly SLR geocenter time series (GCN_RL06_2018_11) from 2002.0 to 2018.9 provided by the University of Texas at Austin Center for Space Research (CSR) (Cheng et al. 2013). These data are determined from the analysis of geodetic satellites LAGEOS-1 and 2, Starlette, Stella and Ajisai observations (Pearlman et al. 2019), for the period 2002.0-2018.9 based on 1-month long satellite orbital arcs. These geocenter time series represent the vector between the SLR Terrestrial Reference Frame (SLRF2014) and the instantaneous mass center of the Earth. The SLRF2014 is a special realization of ITRF2014. In this solution, the gravity field coefficients up to degree and order $5 / 5$ are simultaneously estimated, whereas the Earth rotation parameters and station coordinates are not estimated. For these solutions only the atmosphere and ocean de-aliasing models were used for consistency with the GRACE RL products (Cheng et al. 2013) and non-tidal loading effects were not considered.

\subsection{GNSS Solution}

We employed daily GNSS geocenter time series (5-4_igs.sum) provided by the International GNSS Service (IGS, Ferland and Piraszewski 2009; Rebischung et al. 2016a, b) from 1994.0 to 2019.0. These combined solutions are determined using the seven parameter Helmert transformation between the mean value of the GNSS orbit origins of particular IGS analysis centers and the origin of the IGS14 reference frame, which is the IGS implementation of the ITRF2014. Non-tidal loading effects are not taken into account in these solutions.

\subsection{DORIS Solution}

We use weekly DORIS geocenter time series (ids18wd01.geoc) from 1993.0 to 2018.0 computed by the Combination Center of the International DORIS Service (IDS, Willis et al. 2010). These combined geocenter time series are produced by the IDS based on solutions from individual analysis centers such as Institut Géographique National (IGN), Institute of Astronomy of the Russian Academy of Sciences (INA), and the Space Geodesy Team of the CNES-GRG (LCA, Soudarin and Ferrage 2017). The DORIS geocenter time series are determined using the Helmert transformation between the instantaneous DORIS terrestrial reference frame origin and 
the ITRF2014 origin. In these solutions the non-tidal loading effects were not considered.

\subsection{GRACE and The Ocean Bottom Pressure Model}

The time series of the degree one spherical harmonic coefficients $\left(\mathrm{C}_{10}, \mathrm{C}_{11}, \mathrm{~S}_{11}\right)$ are used to determine the Earth center of mass variations which result from mass redistribution within the Earth's fluid layers. These coefficients are determined by the JPL, California Institute of Technology, GFZ German Research Centre for Geosciences and CSR in the framework of the Tellus GRACE Project, based on K-Band and GPS positions of the gravimetric US and German space agencies (NASA and DLR) twin mission GRACE (Tapley et al. 2004) and GRACEFO (Gravity Recovery and Climate Experiment Follow-On) and the OBP model. The coefficients are provided with a temporal resolution of 1 month.

The time series of the degree one spherical harmonic coefficients cannot be directly determined from gravimetric measurements because the observations provided by the GRACE mission are referred in the frame with the CM origin. In such a frame the degree one spherical harmonic coefficients are equal to zero. Swenson et al. (2008) proposed a method that allows determining degree one geopotential coefficients based on combining GRACE data and oceanic degree one coefficient extracted from the OBP model. However, Swenson et al. (2008) method has a limitation based on the underestimation of the annual amplitude of $\mathrm{C}_{10}$ coefficient, which in turn correspond to the $\mathrm{Z}$ component of geocenter motion. To overcome this problem, Sun et al. (2016, 2017) proposed a modification of Swenson et al. (2008) methodology based on estimation degree one as well as $\mathrm{C}_{20}$ coefficients by the statistically optimal combination of GRACE data and the OBP model. This methodology assumes not only simulating the timevariable gravity fields using synthetic models but also taking into account errors occurring in the real GRACE and the OBP data. As a result, annual variations and long-term trend in degree one and $C_{20}$ coefficients estimations were improved (more details can be found in Sun et al. 2016, 2017).
According to processing standards of the GRACE data, GRACE gravity field coefficients contain the full effects of terrestrial water storage and only remaining atmospheric and oceanic signals. This is due to the fact that GRACE coefficients do not include high-frequency non-tidal atmospheric and ocean mass variability (Swenson et al. 2008). Therefore, the obtained degree one geopotential coefficients represent only mass changes within the land. In order to obtain geocenter coordinates including all information about mass redistributions, also within oceans and the atmosphere, it is necessary to add models representing them. These models are a part of the Atmosphere and Ocean De-Aliasing Level-1B (AOD1B) product and are provided as monthly-means coefficients obtained from the sum of the effect of the atmosphere (ATM) and the dynamic ocean contribution to the OBP (called OCN) (Dobslaw et al. 2017). The ATM represents the contribution of atmospheric surface pressure over the continents, the static contribution of atmospheric pressure to the OBP elsewhere and the much weaker contribution of upper-air density anomalies above both continents and oceans. These models are provided with a 1-month sampling interval as GAC product by the CSR and are obtained from Release-06 GRACE Level-2 (RL06) set (Bettadpur 2018).

Cartesian geocenter coordinates from degree one geopotential coefficients are computed by the formulae (e.g., Cheng et al. 2013; Crétaux et al. 2002; Swenson et al. 2008):

$$
\begin{aligned}
& X=\sqrt{3} R\left(C_{11}^{G R A C E}+C_{11}^{O C N+A T M}\right) \\
& Y=\sqrt{3} R\left(S_{11}^{G R C E}+S_{11}^{O C N+A T M}\right) \\
& Z=\sqrt{3} R\left(C_{10}^{G R A C E}+C_{10}^{O C N+A T M}\right)
\end{aligned}
$$

where $R$ is the mean Earth radius; $C_{10}^{G R A C E}, C_{11}^{G R A C E}, S_{11}^{G R A C E}$ are degree one geopotential coefficients from the GRACE solutions and $C_{10}^{O C N+A T M}, C_{11}^{O C N+A T M}, S_{11}^{O C N+A T M}$ represent respective contributions from models related to the ocean and atmosphere-induced mass redistribution. The geocenter coordinates estimated on the basis of Sun et al. $(2016,2017)$ method (Eq. 1) for the period from August 2002 to February 2015 were used and they are called GRACE + OBP data in this paper. 


\section{Amplitude Spectra of Real- and Complex-Valued Geocenter Coordinates}

To compute the amplitude spectra and to show the variability of amplitudes and phases of oscillations in the geocenter time series, the combination of the Fourier Transform Band Pass Filter (FTBPF) and the Hilbert Transform (HT) method was applied to the real-valued time series $r(n), n=0,1, \ldots, N-1$, representing geocenter variations in each $\mathrm{X}, \mathrm{Y}$ and $\mathrm{Z}$ coordinates (Kosek et al. 2015).

In the FTBPF + HT algorithm, the real-valued broadband oscillation $u(n, \omega) \quad n=0,1, \ldots, N-1$, with central frequency $\omega=2 \pi / T>0$ is used to construct the complex-valued oscillation $z(n, \omega)$ as follows:

$z(n, \omega)=u(n, \omega)+i \cdot H T[u(n, \omega)], \quad n=0,1, \ldots, N-1$

where $H T$ denotes the Hilbert Transform operator, $u(n, \omega)=F T^{-1}[F T(r(n)) \cdot P(\mu, \omega)]$ is the output computed by the FTBPF from real-valued time series $r(n)$ (Kosek 1995) and FT is the Fourier Transform operator. In the FTBPF algorithm the parabolic transmittance function was applied:

$$
P(\mu, \omega)=\left\{\begin{array}{l}
1-\left(\frac{\mu-\omega}{\lambda}\right)^{2} \\
0
\end{array}\right\} \begin{array}{ll}
\text { for } & |\mu-\omega| \leq \lambda ; \\
\text { for } & |\mu-\omega|>\lambda ;
\end{array}
$$

in which $\omega=2 \pi / T>0$ and $\lambda>0$ is the spectral window half-width that satisfies the condition $\omega-\lambda \geq 0, T$ is the central period of the oscillation, $\mu$ is the frequency argument.

It can be proved that the complex-valued oscillation (Eq. 2) is expressed by the following formula (Gasquet and Witomski 1999; Popiński 2008):

$$
z(n, \omega)=F T^{-1}[F T(r(n)) \cdot P(\mu, \omega)(\operatorname{sign}(\mu)+1)] .
$$

The discretized formula representing the above equation reads:

$z(n, \omega) \frac{2}{N} \sum_{v=0}^{N / 2} \tilde{r}(v) P(2 \pi v / N, \omega)(1+\operatorname{sign}(\mu)) \exp (i 2 \pi v n / N)$, where $N$ is even, $n=0,1, \ldots, N-1$, and $\tilde{r}(v)$, $v=-N / 2+1,-N / 2+2, \ldots, N / 2$, represent the Discrete Fourier Transform (DFT) coefficients corresponding to the analyzed series $r(n)$. The variable amplitudes and phases of the oscillation $z(n, \omega)$ with central frequency $\omega$ are computed by:

$$
\begin{aligned}
& A(n, \omega)=\sqrt{[\Re(z(n, \omega))]^{2}+[\Im(z(n, \omega))]^{2}}, \\
& \varphi(n, \omega)=\arctan \left[\frac{\Im(z(n, \omega))}{\Re(z(n, \omega))}\right] \frac{2 \pi n \Delta t}{T},
\end{aligned}
$$

where $\Delta t$ is the sampling interval.

The mean amplitudes (also the mean amplitude spectra of real-valued time series) and phases of the oscillation are computed by the following formulae:

$$
\begin{gathered}
A_{m}(\omega)=\frac{1}{N-2 n_{f}} \sum_{n_{f}+1}^{N-n_{f}} A(n, \omega), \\
\varphi_{m}(\omega)=\frac{1}{N-2 n_{f}} \sum_{n_{f}+1}^{N-n_{f}} \phi(n, \omega)
\end{gathered}
$$

where $n_{f}$ is the number of points to be dropped at the end and at the beginning of the filtered time series due to filtering errors at boundaries.

To compute the complex-valued oscillation with central frequency $\omega$ from the complex-valued geocenter time series $s(n)=\left\{\begin{array}{l}x(n)+i y(n) \\ y(n)+i z(n) \\ z(n)+i x(n)\end{array} \quad n=0,1, \ldots, N-1\right.$ the

FTBPF is applied:

$$
g(n, \omega)=F T^{-1}[F T(s(n)) \cdot P(\mu, \omega)]
$$

The mean FTBPF amplitude spectra of prograde $(\omega>0)$ and retrograde $(\omega<0)$ oscillations are computed by the following formula (Kosek 1995):

$$
A_{s}(\omega)=\sqrt{\frac{2}{N-2 n_{f}} \sum_{n_{f}+1}^{N-n_{f}}|g(n, \omega)|^{2}}
$$

\subsection{Annual and Semi-Annual Oscillations of Geocenter Coordinates}

In geocenter time series, the dominant oscillations are the annual and semi-annual ones. The annual oscillation results from the seasonal mass redistribution within the atmosphere, ocean and continental hydrology effecting on geocenter motion (Dong et al. 
2014). Thus, the goal of the manuscript is to determine geocenter motion model in which annual and semi-annual oscillations are present. In the manuscript, other shorter period oscillations with periods less-equal than 112 days were not considered, because they are filtered out by adding only 6 the WBSF frequency components with the lowest frequencies. The Lagrange interpolating polynomial of degree 2 was applied, to interpolate daily GNSS and monthly GRACE + OBP and SLR geocenter coordinates with 1-week sampling interval. The computation methods used in this paper need equidistant time series and 1-week sampling interval represents a median sampling interval of all geocenter time series. Next, the FTBPF + HT was applied to compute variable amplitudes of the annual and semi-annual oscillations in the interpolated geocenter time series. The $\lambda=0.014$ parameter of the parabolic transmittance function was selected in such a way to optimally separate the annual and semi-annual oscillations. Decrease of this parameter cause variable amplitudes and phases (Eq. 6) to be flatter and the mean values of amplitudes become smaller. The amplitudes of the annual and semi-annual oscillations computed by the FTBPF + HT (Eq. 6) in geocenter coordinates determined by DORIS, GNSS, GRACE + OBP, and SLR techniques vary in time (Figs. 1, 2). The annual oscillation amplitude in the geocenter coordinates is variable because it can be caused by real geocenter motion and systematic signals of the annual character due to mis-modeling of satellite orbits, which can be different in different techniques. In the $\mathrm{X}$ and $\mathrm{Y}$ components, the amplitudes of the annual oscillation change from 0 to $5 \mathrm{~mm}$ and the semi-annual oscillation amplitude is about two times smaller than the annual oscillation one. In the case of the $\mathrm{Z}$ component, the variations of amplitudes of these two oscillations are larger than for the $\mathrm{X}$ and $\mathrm{Y}$ components, except for the GRACE + OBP solution where it is of the same order as for the equatorial components. There is no agreement in the time-varying amplitudes between different techniques due to systematic errors in these techniques, the character of which can be annual. The amplitude of the annual signal in the $\mathrm{Z}$ component determined from the GNSS solution decreases from 8
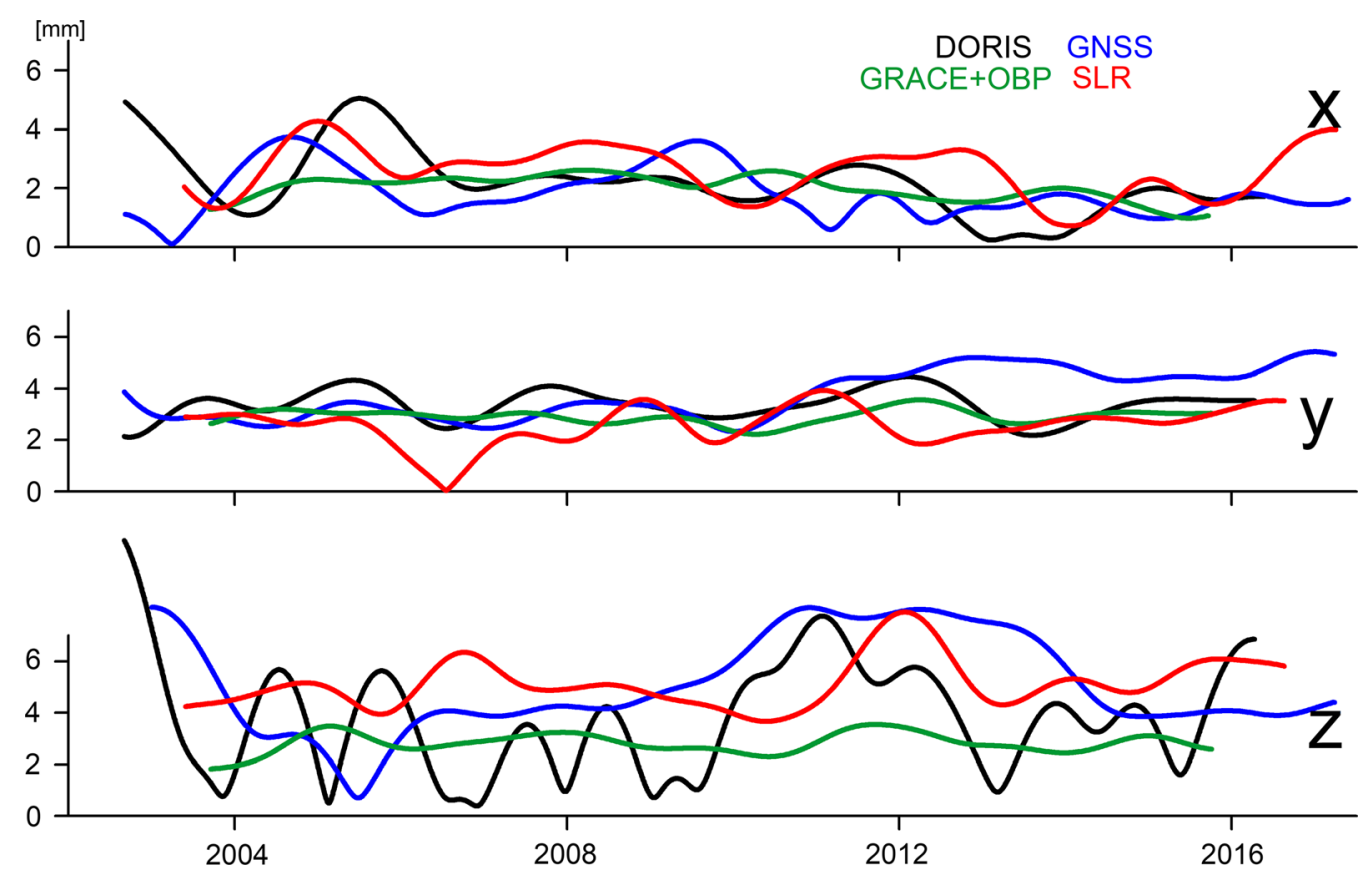

Figure 1

The amplitudes of the annual oscillation from the SLR, GNSS, DORIS, and GRACE + OBP geocenter coordinates, determined using the FTBPF + HT combination for filter window halfwidth $\lambda=0.014$ 

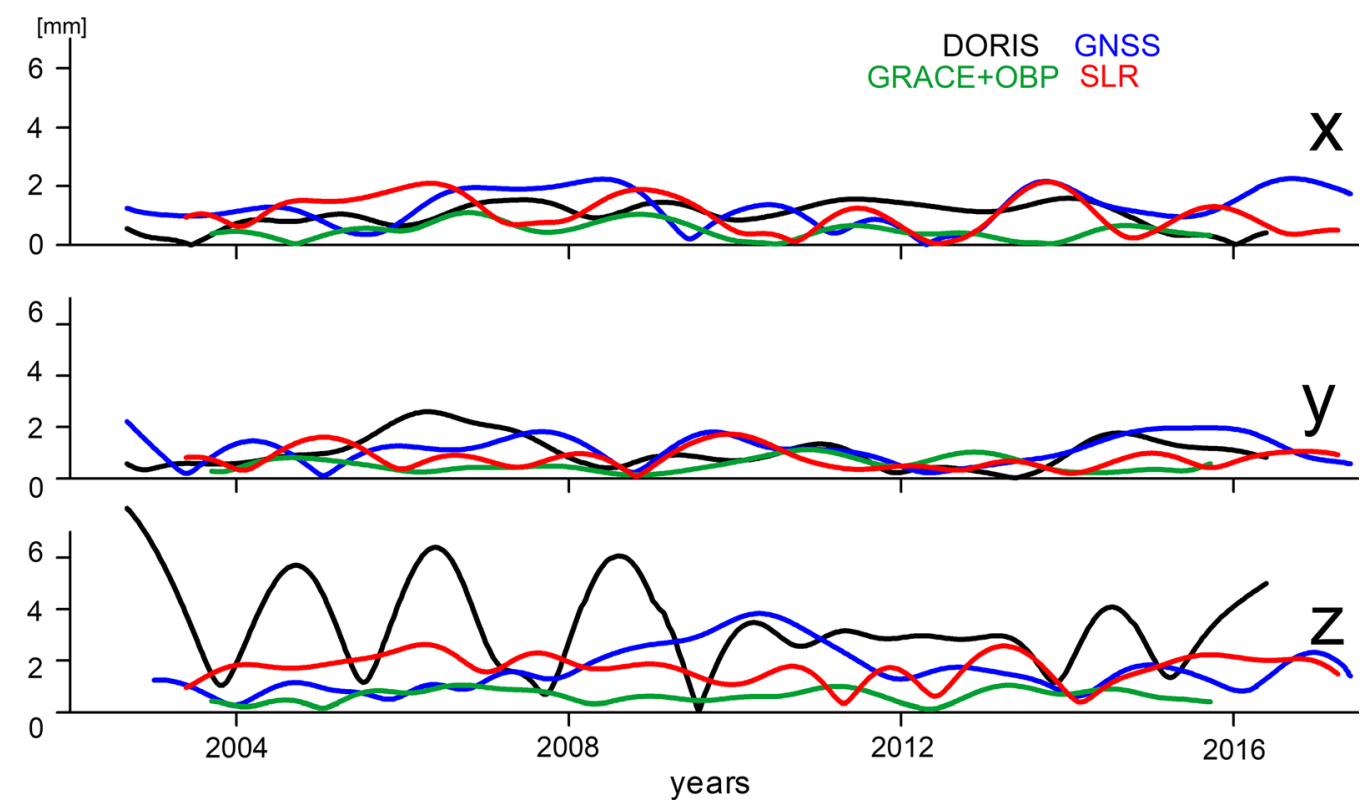

Figure 2

The amplitudes of the semi-annual oscillation from the SLR, GNSS, DORIS, and GRACE + OBP geocenter coordinates, determined using the FTBPF + HT combination for filter window halfwidth $\lambda=0.014$

to $1 \mathrm{~mm}$ between 2003 and 2005 and then increases from about 4 to $7 \mathrm{~mm}$ in 2005-2010, which can possibly be associated with the increasing contribution of GLONASS satellites to this solution. The amplitudes of the annual oscillation in the $\mathrm{Z}$ component for the DORIS series show large variations ranging from 0.5 to $7.0 \mathrm{~mm}$.

The phases of the annual oscillation computed using the FTBPF + HT for all the considered techniques change in time and generally agree for the X component of GNSS and SLR series and for the Y component of DORIS and SLR series (Fig. 3). In the case of the GRACE + OBP technique, the phases of the annual oscillation are almost constant in all components. From 2005 to 2014 the phase of the annual oscillation of DORIS technique in the $\mathrm{X}$ component is closer the phase of the annual oscillation in GRACE + OBP data and after 2013 it decreases and reaches the phase values of the annual oscillations in GNSS and SLR data. In the Z component, the phases of the annual oscillations are close for the SLR and GNSS techniques. The phase of the annual oscillation in the $\mathrm{Z}$ component of the DORIS series shows large variations. Geocenter coordinates derived from DORIS are typically affected by solar radiation pressure modeling issues which generate spurious signals in the $\mathrm{Z}$ component with the dominating periods of 118 days and 1 year (Gobinddass et al. 2009).

The phases of the semi-annual oscillation show larger variations than those of the annual oscillation and their values fluctuate in the entire phase range $[0$, $360^{\circ}$ ]. There is no agreement between phase variations of this oscillation for different techniques due to the smaller amplitude of the semi-annual oscillation than the annual one, as well as random and systematic errors in these techniques' geocenter coordinates (Fig. 4).

Averaging of variable amplitudes and phases of the annual and semi-annual oscillations in the common time span 2004.6-2015.6 of all techniques enables the computation of the mean values as shown in Table 1. The mean errors of constant amplitudes and phases were computed as standard deviations of the differences between their variable and mean values. This time span used for the comparison is reduced by subtracting 500 days in the beginning and at the end of the filtered series in order to remove 

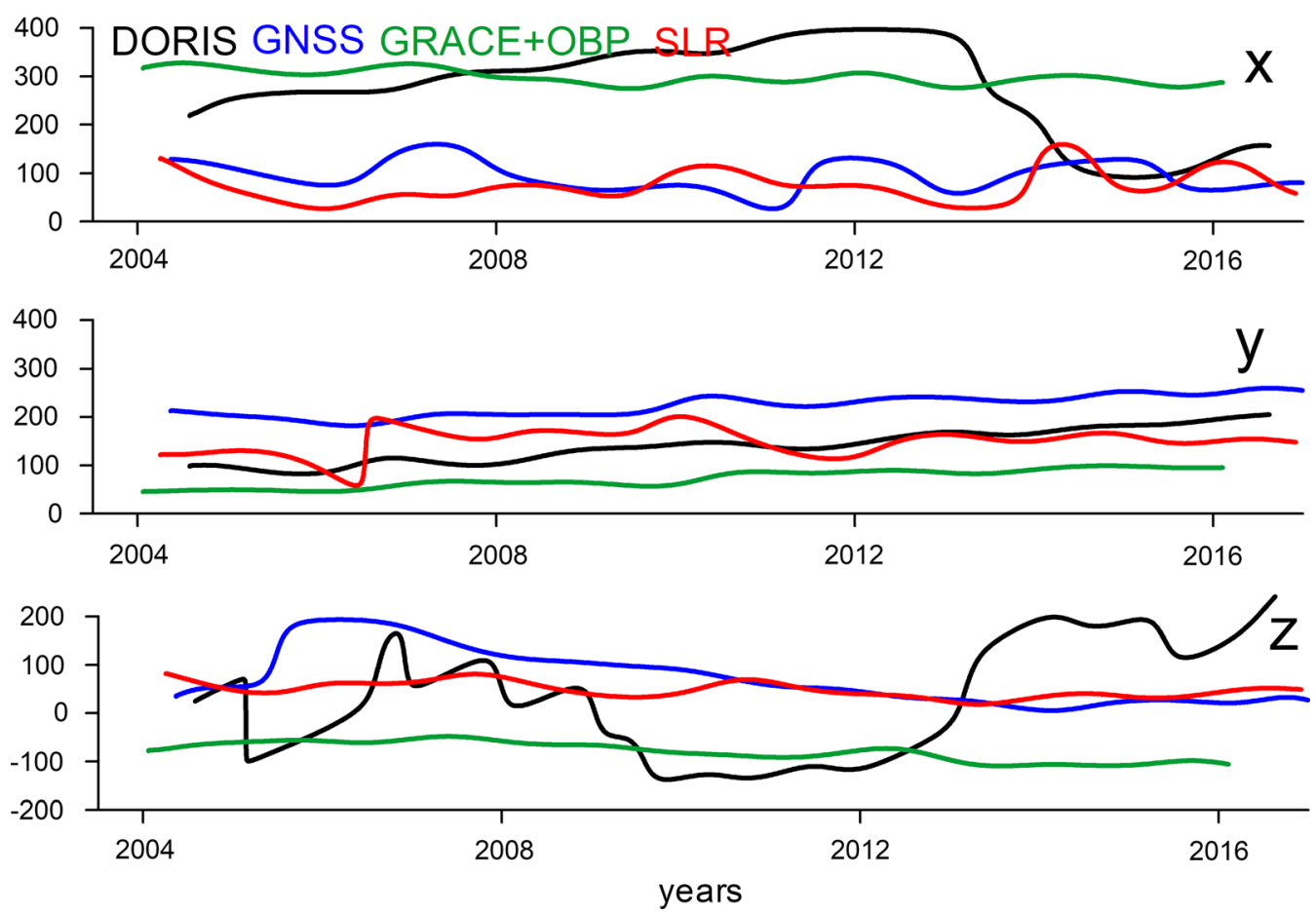

Figure 3

The annual oscillation phase variations in the SLR, GNSS DORIS and GRACE + OBP geocenter coordinates, determined by the FTBPF + HT combination for filter window halfwidth $\lambda=0.014$. Zero phase crossings for the SLR, GNSS, GRACE + OBP, and SLR solutions correspond to the same epoch 2004.6

high filtering errors at boundaries. The mean amplitudes of the annual oscillation are of the same order of about $2 \mathrm{~mm}$ for the $\mathrm{X}$ component from all techniques. The mean amplitudes of the annual oscillation in the $\mathrm{Y}$ component are larger than for the $\mathrm{X}$ one and are of the order of 2.4-3.6 $\mathrm{mm}$. In the case of the $\mathrm{Z}$ component, the amplitude of the annual oscillation is of the order of $2.8-3.6 \mathrm{~mm}$ for the GRACE + OBP and DORIS series and 5.1-5.6 mm for the SLR and GNSS series.

Generally, the amplitudes of the annual oscillation are of the same order as those obtained by other authors. For the $\mathrm{Z}$ component, the amplitudes are usually larger by about $2 \mathrm{~mm}$ than for the $\mathrm{X}$ or $\mathrm{Y}$ components, except for GRACE + OBP from this study, where they are of the same order as for the equatorial X, Y components. The mean amplitudes of the annual oscillation in the $\mathrm{X}$ component are of the same order of $2 \mathrm{~mm}$ for all the techniques and agree well with those from Swenson et al. (2008). The mean amplitudes of the annual oscillation in the $\mathrm{Y}$ component from the DORIS and GNSS solutions are larger by about $1 \mathrm{~mm}$ than in the case of the SLR and GRACE + OBP results. The mean amplitudes of the annual oscillation from the GRACE + OBP solutions agree well with those from Swenson et al. (2008) for $\mathrm{X}$ and $\mathrm{Y}$ components and for $\mathrm{Z}$ component the annual amplitude is higher by $1 \mathrm{~mm}$ which is consistent with Sun et al. (2017). The mean amplitudes of the annual oscillation in the $\mathrm{Z}$ component in the SLR and GNSS solutions agree well with that from Li et al. (2012) and Glaser et al. (2015a, b) and achieve the level of about $5 \mathrm{~mm}$ (see Table 1).

The mean amplitudes of the annual oscillation in all components of the SLR series agree well with the results reported by Altamimi et al. (2016) for ITRF2014, whereas the $\mathrm{Z}$ coordinate in DORIS solution disagrees with ITRF2014. The annual and semi-annual oscillation phases together with the amplitudes are necessary to construct a deterministic model. However, the mean phases in this study, are referred to different epochs thus, their values shown 


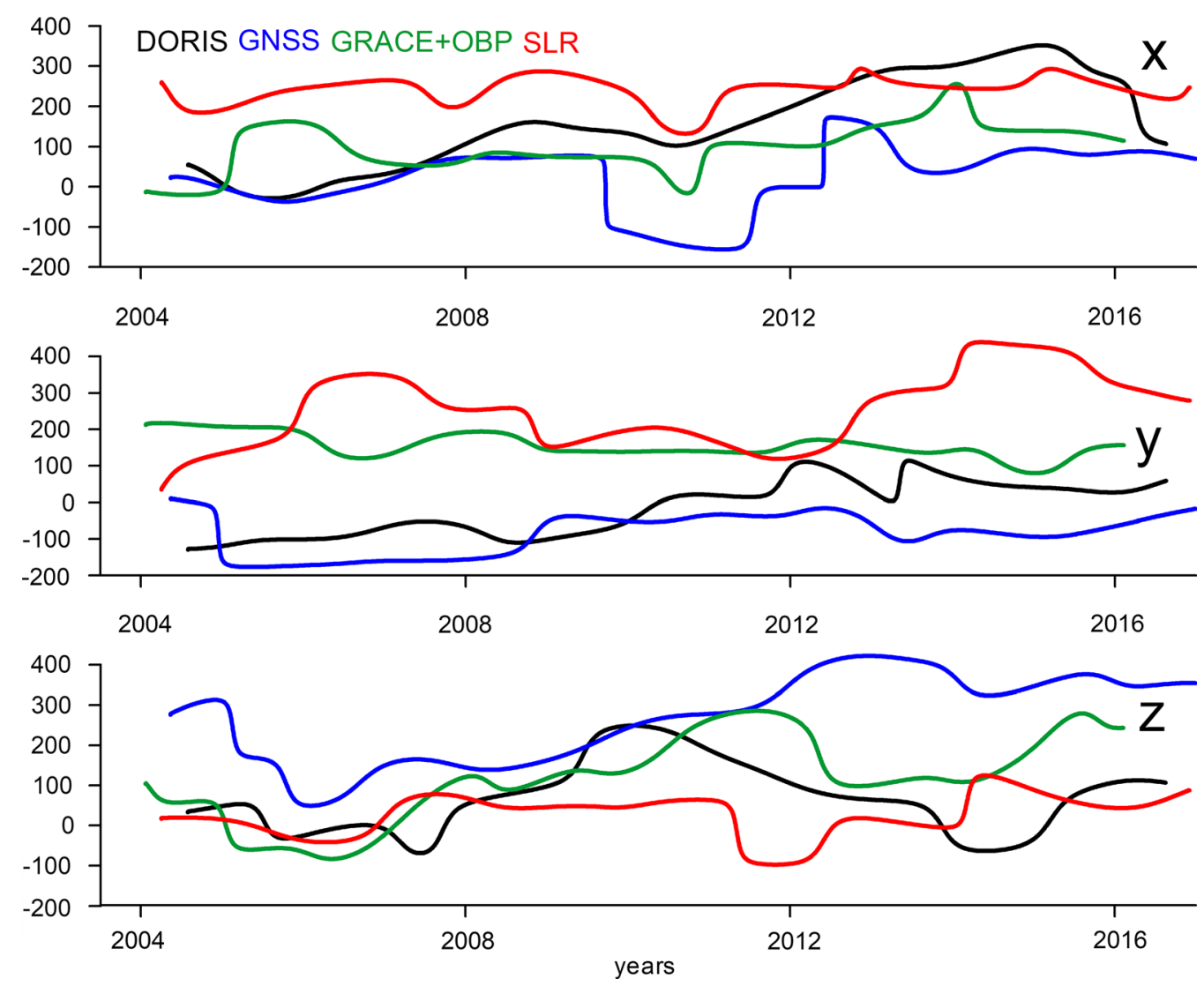

Figure 4

The phase variations of the semi-annual oscillation from the SLR, GNSS, DORIS, and GRACE + OBP geocenter coordinates, determined using the FTBPF + HT combination for filter window halfwidth $\lambda=0.014$. Zero phase crossings for the SLR, GNSS, DORIS, and GRACE + OBP solutions correspond to the same epoch 2004.6

in Table 1 cannot be compared with the values obtained by other authors. Moreover, due to high variations of the semi-annual oscillation phases in the entire phase range $\left[0^{\circ}, 360^{\circ}\right]$ (Fig. 4) their mean values (Table 1) do not seem to be meaningful because the computed standard deviations of phase variations are very close to the standard deviation of the uniform distribution given by the formula $\sigma=\left(360^{\circ}-0\right) / \sqrt{12} \approx 104^{\circ}$.

\section{Stochastic Model of the Geocenter Motion}

The WBSF (Cooper 2009) with the discrete Shannon wavelet functions (Frazier and Torres 1994) is used to generate the common geocenter motion model based on DORIS, GNSS, GRACE + OBP, and SLR data. This kind of the WBSF, which enables detecting common oscillations in two complex-valued time series, was applied for the first time to geocenter time series by Kosek et al. (2014) to construct the geocenter motion model based on the GNSS geocenter time series from the IGS Analyses Centres and the SLR geocenter series generated using the Bernese GNSS Software (Dach et al. 2015).

In this paper, the WBSF is applied to detect common signals in two real-valued time series assuming the threshold value equal to 0.9 . Such a threshold value is interpreted as the cosine of a threshold angle between wavelet transform coefficients vectors corresponding to oscillations with different frequencies. That is why common oscillations in the two time series are those which have similar phases. The number of input data points for the WBSF should be equal to 


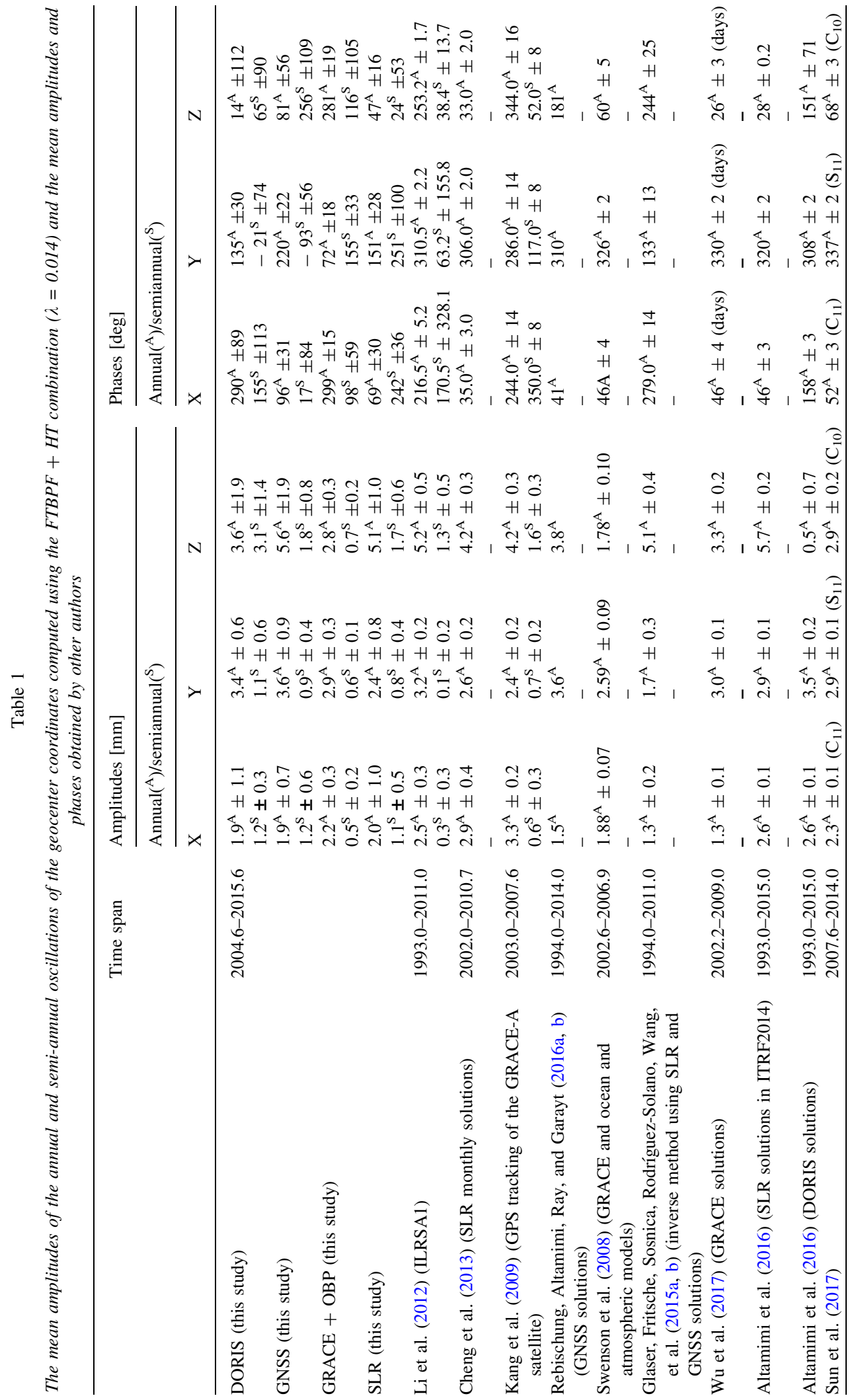




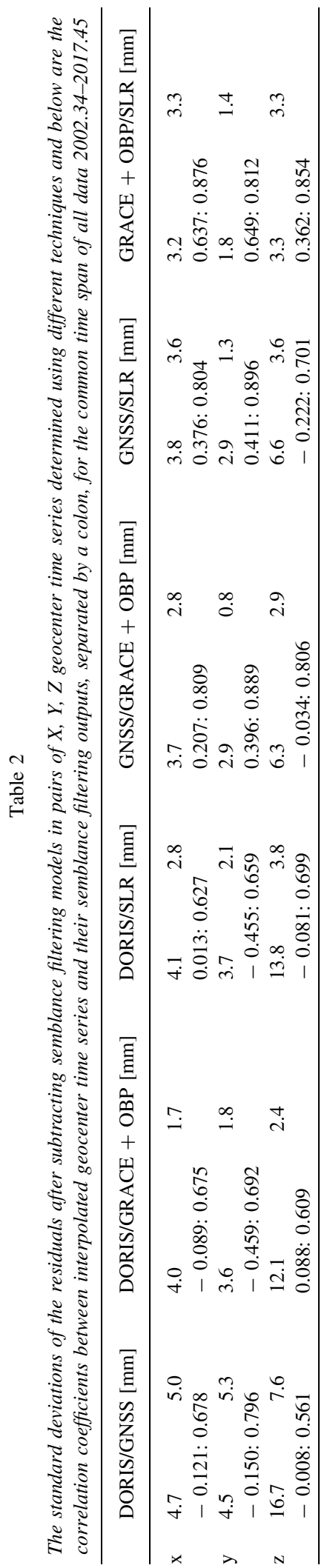

$N=2^{p}$, where $p$ denotes the maximal number of frequency components. Instead of cutting the number of data so that they fulfill this condition, they were forecasted backward and forward by the autocovariance prediction (Kosek 2002) so as to create a new time series with a number of points equal to $N$. In the autocovariance prediction forecast method the first prediction point of stationary time series is computed in such a way that the sum of squares of the differences between autocovariance estimation of time series and the autocovariance estimation of the same time series extended by the prediction point is minimum. After the first prediction point is computed it is added as the last time series point so the next prediction point can be computed. The number of frequency components depends on the number of common data $N_{c}$ in the two chosen interpolated geocenter time series. The value $p$ should satisfy the following condition $2^{p-1}<N_{c}<2^{p}$. Thus, in the case of the interpolated GRACE + OBP and other interpolated geocenter time series with 1week sampling interval the number of common points is equal to $N_{c}=789$, so $p=10$. Each WBSF output was constructed as the sum of only the 6 lowest frequency components. When the normalized frequencies of the Shannon wavelet functions used to construct the outputs are equal to $f_{k}=2^{k} / 2^{p}$ for $k=0,1, \ldots, p-1$, then the 4 highest frequency components with the indices $k=p-4, p-$ $3, \ldots, p-1$ were not included in these outputs. These normalized frequencies are equal to $1 / 2,1 / 4,1 / 8$ and $1 /$ 16, which for the sampling interval $\Delta t=7$ days, correspond to oscillations with periods of 14, 28, 56 and 112 days, respectively. Thus, for this assumed sampling interval all oscillations with periods less-equal than 112 days were not included in the stochastic geocenter motion model. The number of common points of the interpolated DORIS and GNSS time series was $N_{c}=1251$, and in this case, the initial values of both time series were rejected so that the common number of points is $N_{c}=N=1024$, so $p=10$. Next, the beginning and the end part of the frequency components computed by the WBSF method corresponding to these forecasts were rejected, which allowed a partial reduction of filtering errors at boundaries. The advantage of using the WBSF is the computation of two filtered time series from the input ones, which may have common signals in all 
frequency bands of the input time series. The first output is the first filtered series, in which oscillations are similar to those in the second series, while the second output is the second filtered series, in which oscillations are similar to those in the first series. The remaining signals coming from the differences between the original data and these common signals can be associated with random and systematic errors in each technique. As shown by Rebischung et al. (2013) the sensitivity of GNSS solutions to the geocenter recovery is very limited, in particular for the $\mathrm{Z}$ geocenter component, due to strong correlations between clock parameters, troposphere delays, orbit parameters, and geocenter coordinates. However, as shown by Meindl et al. (2013, 2015), Arnold et al. (2015) and Haines et al. (2015), the recovery of geocenter motion using GPS and GLONASS data is feasible, when using careful and proper modeling of GNSS satellite orbits. The systematic errors in geocenter time series may also be caused by mis-modeling of the solar radiation pressure. Systematic errors largely related to mismodeling of solar radiation pressure also occur in DORIS data, especially in the $\mathrm{Z}$ component of geocenter motions (Gobinddass et al. 2009). In the case of SLR, the modeling of solar radiation pressure is much easier for SLR spherical satellites and its influence is not so significant as in the case of other techniques. However, depending on the satellite technique systematic errors caused by solar radiation pressure have different frequencies and amplitudes thus, they should not be present in the WBSF models, instead, they may occur in the remaining signals of each technique.

The common signals are computed by pairing real-valued $\mathrm{X}, \mathrm{Y}$ and $\mathrm{Z}$ geocenter coordinates in the following pairs of techniques: DORIS/GNSS, DORIS/GRACE + OBP, DORIS/SLR, GNSS/ GRACE + OBP, GNSS/SLR, and GRACE + OBP/ SLR. Next, the weighted mean of common signals

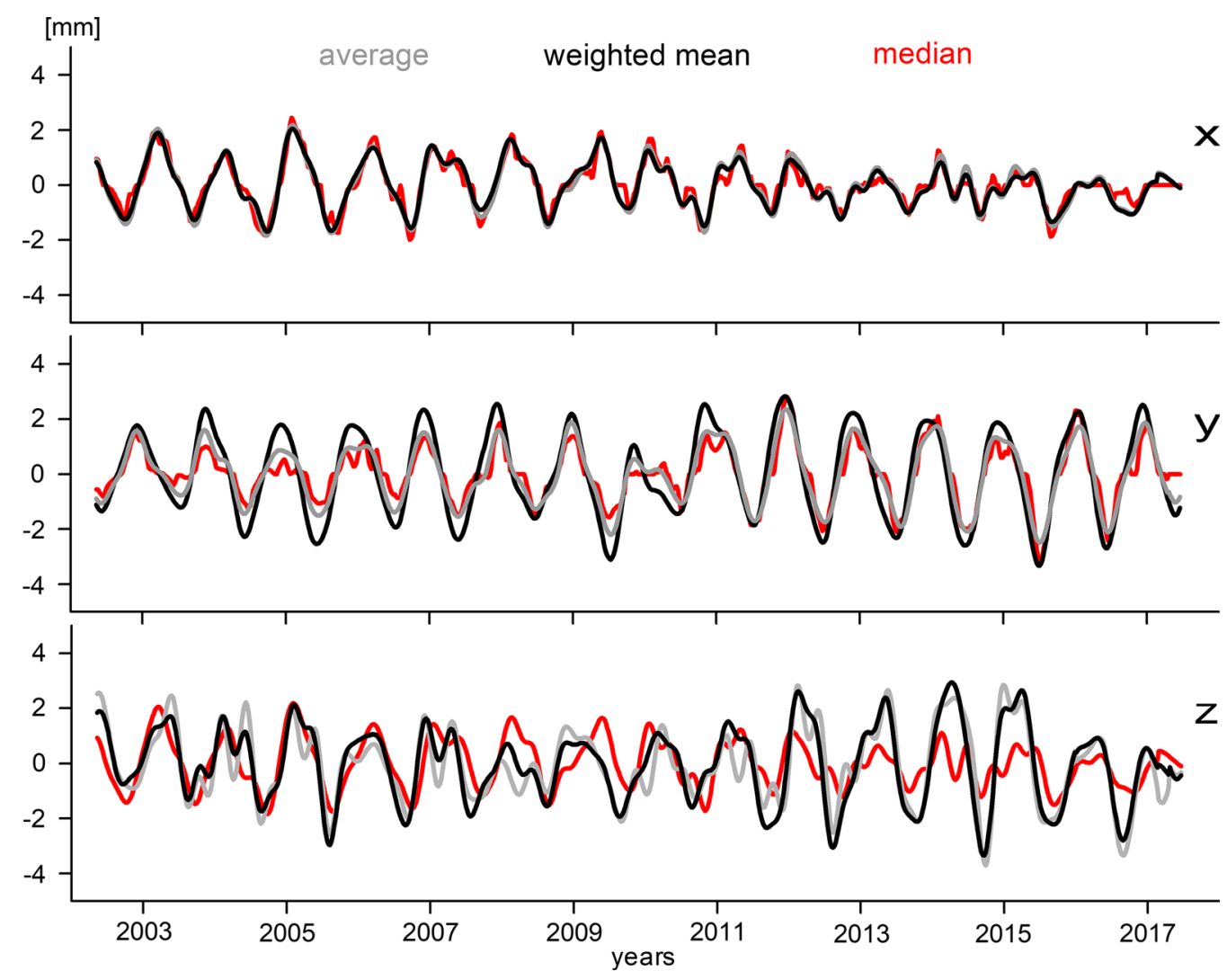

Figure 5

Stochastic models of geocenter motions computed as weighted mean (black), average (grey) and median (red) from the outputs obtained by the WBSF 

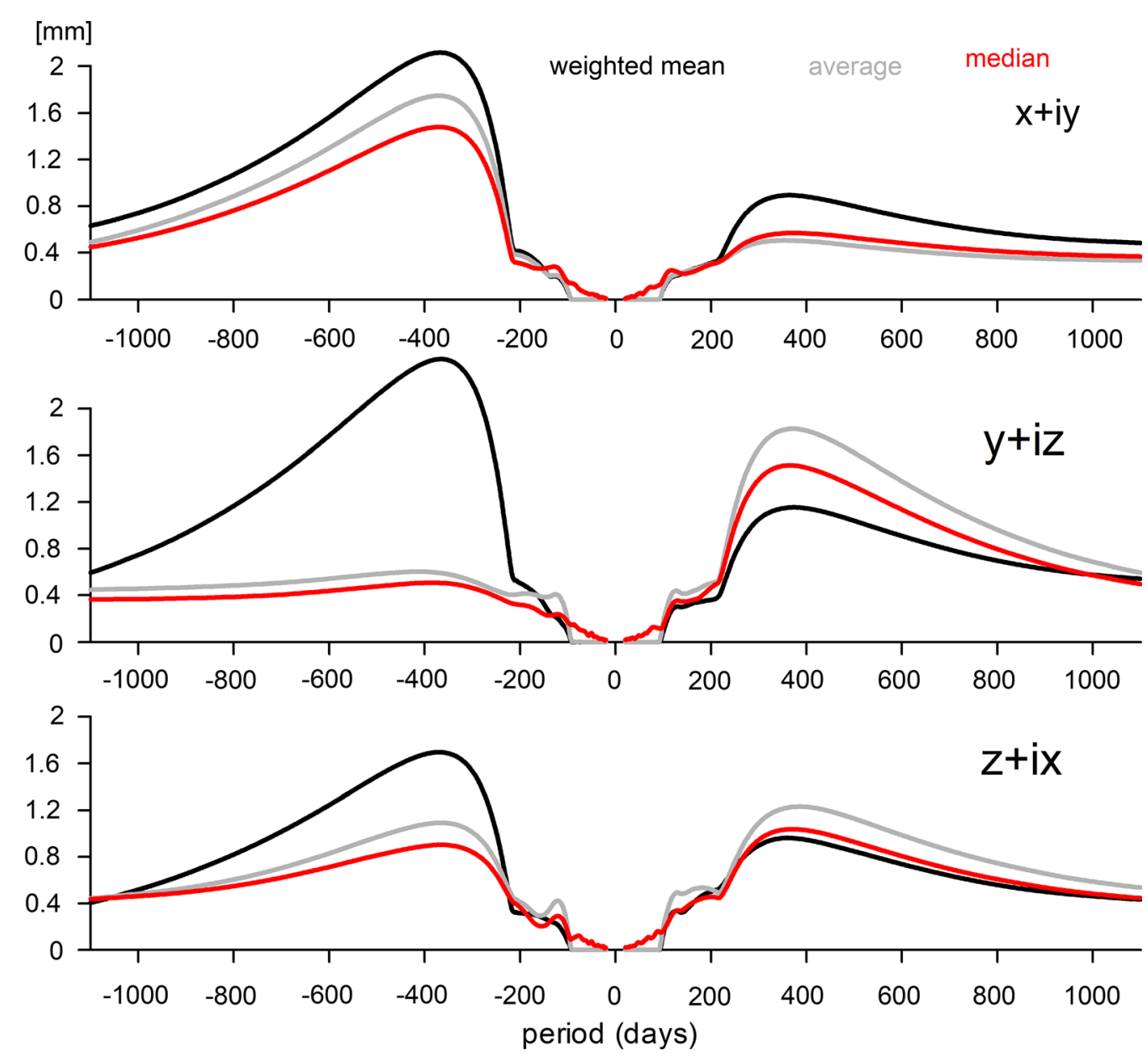

Figure 6

The FTBPF amplitude spectra of complex-valued $\mathrm{X}+\mathrm{iY}, \mathrm{Y}+\mathrm{iZ}$ and $\mathrm{Z}+\mathrm{iX}$ geocenter motion models computed as the weighted mean (black), average (grey), and median (red) shown in Fig. 5

was computed from 12 WBSF outputs assuming weights as inversely proportional to the variances of differences between the geocenter time series and their corresponding common filtered signals. The standard deviations, corresponding to these variances, of the residuals after subtracting semblance filtering outputs in pairs of geocenter time series determined by different techniques in the common time span of all techniques are shown in Table 2. To show the similarity between filtered oscillations in pairs of geocenter time series the correlation coefficients between the semblance filtering outputs were computed (Table 2). These correlation coefficients are larger for the pairs which consist of GNSS, SLR, and GRACE + OBP techniques than for the pairs including DORIS technique. The reason of these smaller, or sometimes negative, correlation coefficients for the pairs including DORIS technique (Table 2) can be e.g. quite significant change in the annual oscillation phase of the order of $300^{\circ}$ in the $\mathrm{X}$ coordinate in 2013-2015 (Fig. 3) and high variations of the annual oscillation phase in the $\mathrm{Z}$ coordinate for the DORIS technique (Fig. 3). Additionally, the average and median models were computed from 12 outputs computed by the WBSF for each coordinate. The comparison of the stochastic geocenter motion models computed using the WBSF outputs shows a dominating annual oscillation in each model. The weighted mean model is more smoothed than the geocenter motion models computed using average 


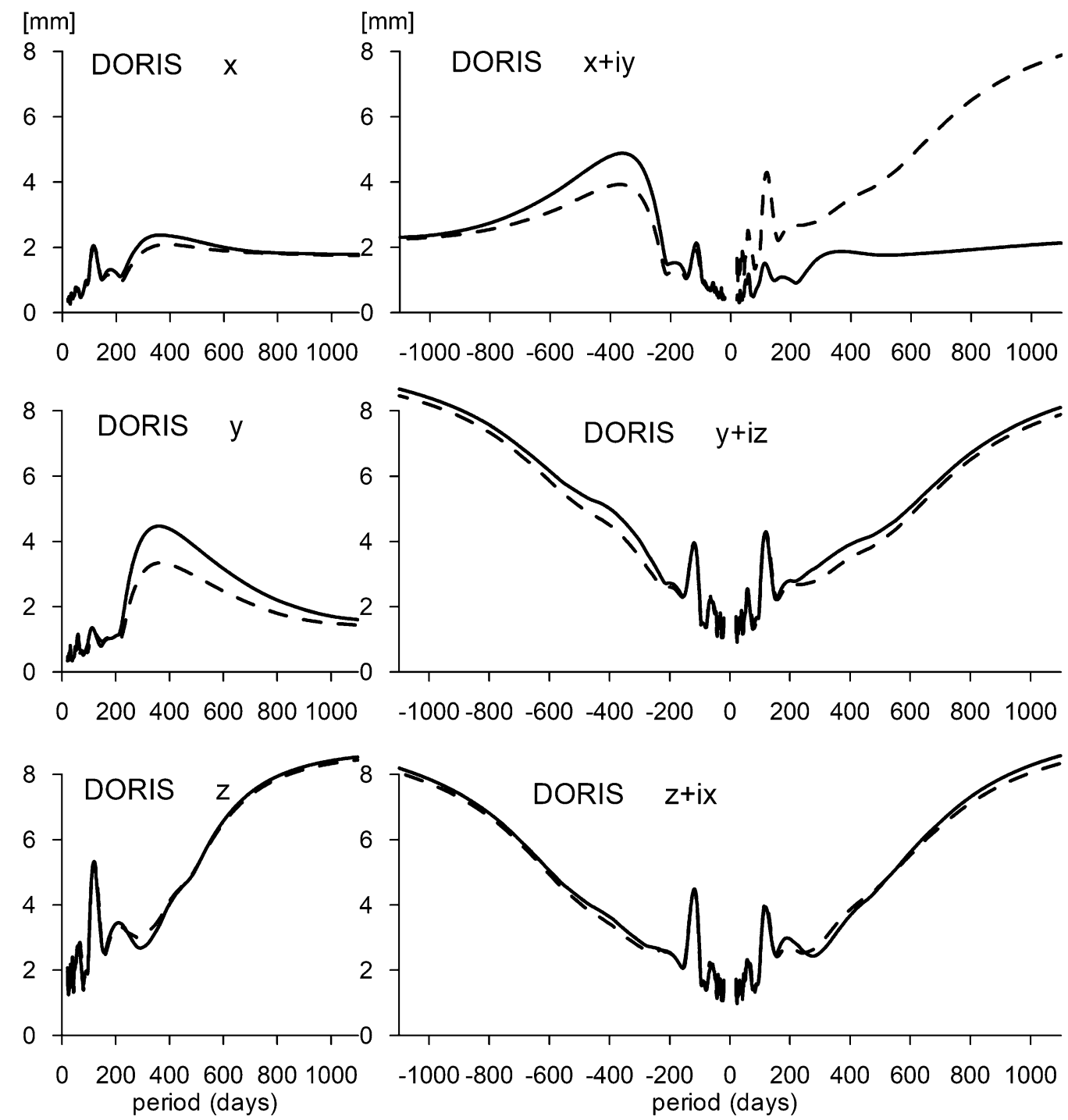

Figure 7

The FTBPF + HT amplitude spectra (left column) of real-valued and the FTBPF amplitude spectra (right column) of complex-valued $\mathrm{X}+\mathrm{iY}, \mathrm{Y}+\mathrm{iZ}$ and $\mathrm{Z}+\mathrm{iX}$ DORIS geocenter coordinates (dashed lines) and DORIS geocenter residuals after subtracting the weighted mean geocenter model (solid lines)

and median values (Fig. 5). Amplitudes and phases of the annual signal agree well in these three stochastic models, especially for the $\mathrm{X}$ coordinate. In the case of the $\mathrm{Y}$ coordinate, the phase agrees moderately well and the amplitude of the annual oscillation in the median and average models are a little smaller than in the weighted mean model. Generally, the average amplitudes of the annual signal are of the order of $2 \mathrm{~mm}$ in each geocenter coordinate. The median model shows smaller amplitude variations than the average and weighted mean models in 2012-2016 in the $\mathrm{Z}$ component.

\section{Spectral Analysis of The Geocenter Coordinates, Their Stochastic Models and Residuals}

To detect polarizations of the annual oscillations present in the geocenter motion models the FTBPF amplitude spectra of complex-valued $\mathrm{X}+\mathrm{iY}$, 

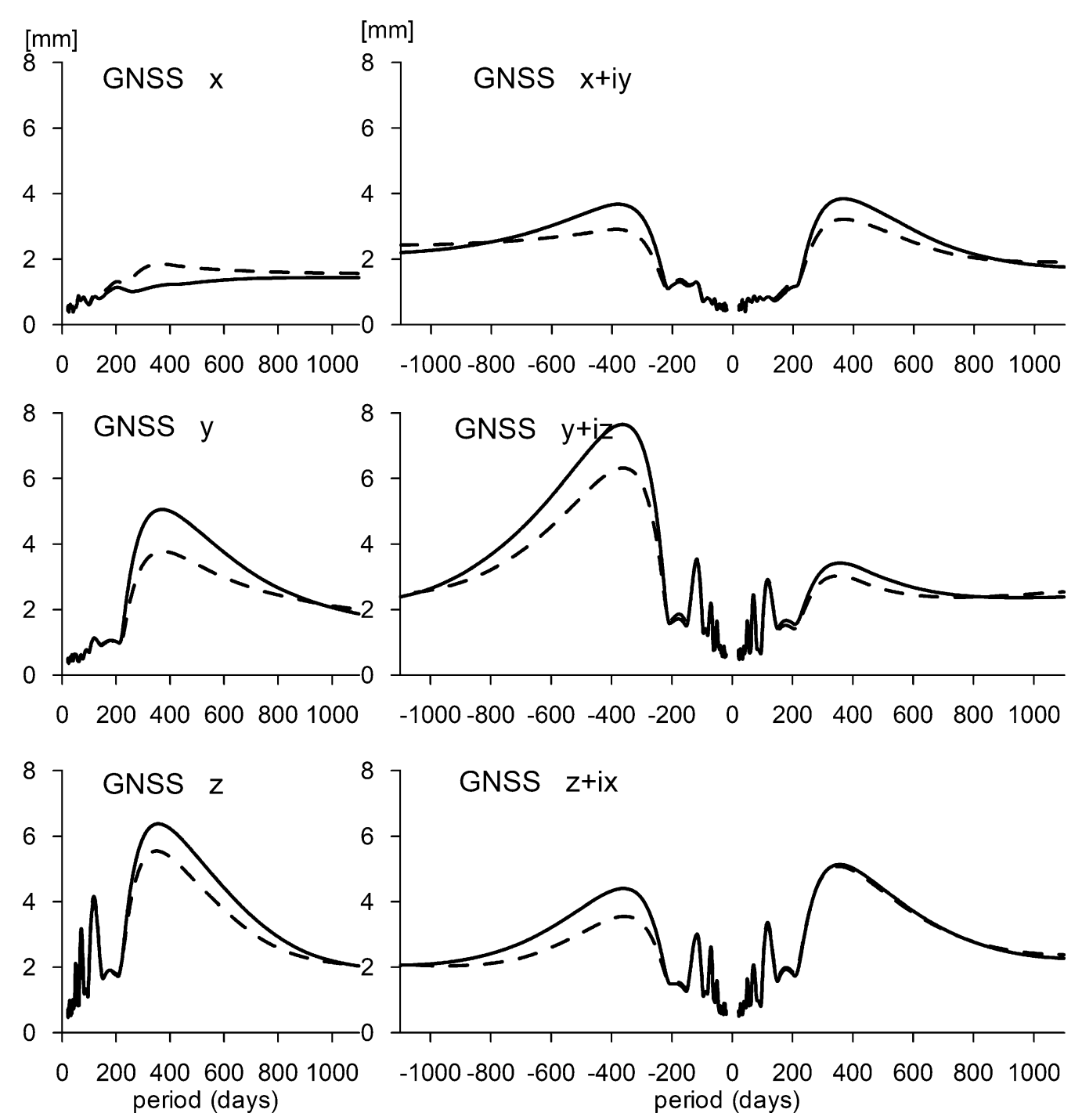

Figure 8

The FTBPF + HT amplitude spectra (left column) of real-valued and the FTBPF amplitude spectra (right column) of complex-valued $\mathrm{X}+\mathrm{iY}, \mathrm{Y}+\mathrm{iZ}$ and Z $+\mathrm{iX}$ GNSS geocenter coordinates (dashed lines) and GNSS geocenter residuals after subtracting the weighted mean geocenter model (solid lines)

$\mathrm{Y}+\mathrm{iZ}$, and $\mathrm{Z}+\mathrm{iX}$ coordinates are computed (Fig. 6). In the case of $X Y$ equatorial plane, the annual signal is mostly retrograde (clockwise) because the peaks in the amplitude spectra for negative periods are larger than for positive ones. The annual signal for the average and median models seems to be more prograde than retrograde while the weighed mean model is more retrograde than prograde for the YZ plane. The annual signal for the weighted mean model is more retrograde than prograde and for the average and median models the prograde and retrograde components of the annual signal are of the same order in the ZX plane.

The FTBPF + HT and the FTBPF amplitude spectra of DORIS, GNSS, GRACE + OBP, and SLR real and complex-valued geocenter time series and their residuals are computed to check the reliability of the weighted mean stochastic geocenter motion model (Figs. 7, 8, 9, 10). The mean amplitude FTBPF spectrum of the complex-valued time series is 

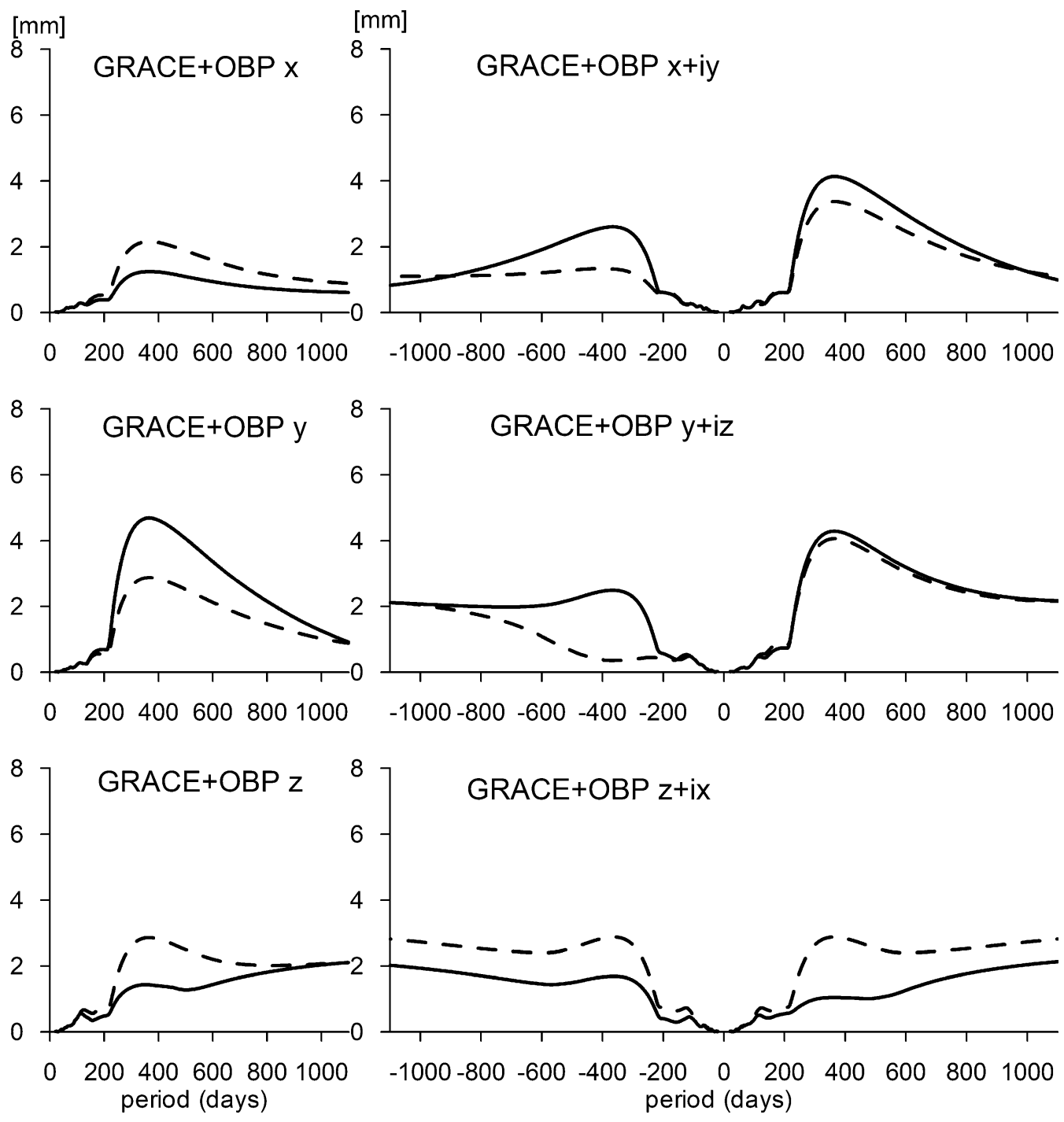

Figure 9

The FTBPF + HT amplitude spectra (left column) of real-valued X, Y, Z and the FTBPF amplitude spectra (right column) of complex-valued $\mathrm{X}+\mathrm{iY}, \mathrm{Y}+\mathrm{iZ}$ and $\mathrm{Z}+\mathrm{iX}$ GRACE $+\mathrm{OBP}$ geocenter coordinates (dashed lines) and GRACE + OBP geocenter residuals after subtracting the weighted mean geocenter model (solid lines)

proportional to the amplitudes of prograde and retrograde oscillations, while the mean amplitude FTBPF + HT spectrum is proportional to the amplitudes of oscillations in each coordinate. The peaks in the FTBPF and FTBPF + HT amplitude spectra corresponding to the annual period are usually higher for GRACE + OBP geocenter coordinates than for the residuals which means that the weighted mean geocenter motion model is very similar to GRACE + OBP data (Fig. 9). The amplitudes of the annual oscillation in the weighted mean models are different from the amplitudes of annual oscillations in DORIS, SLR and GNSS geocenter time series because of the system-specific systematic errors in geocenter coordinates of each technique, e.g. draconitic signal of 352 days in the GNSS series.

In most cases, the amplitude spectra of the residuals in the vicinity of the annual period show different values than the amplitude spectrum of the geocenter time series for the corresponding 

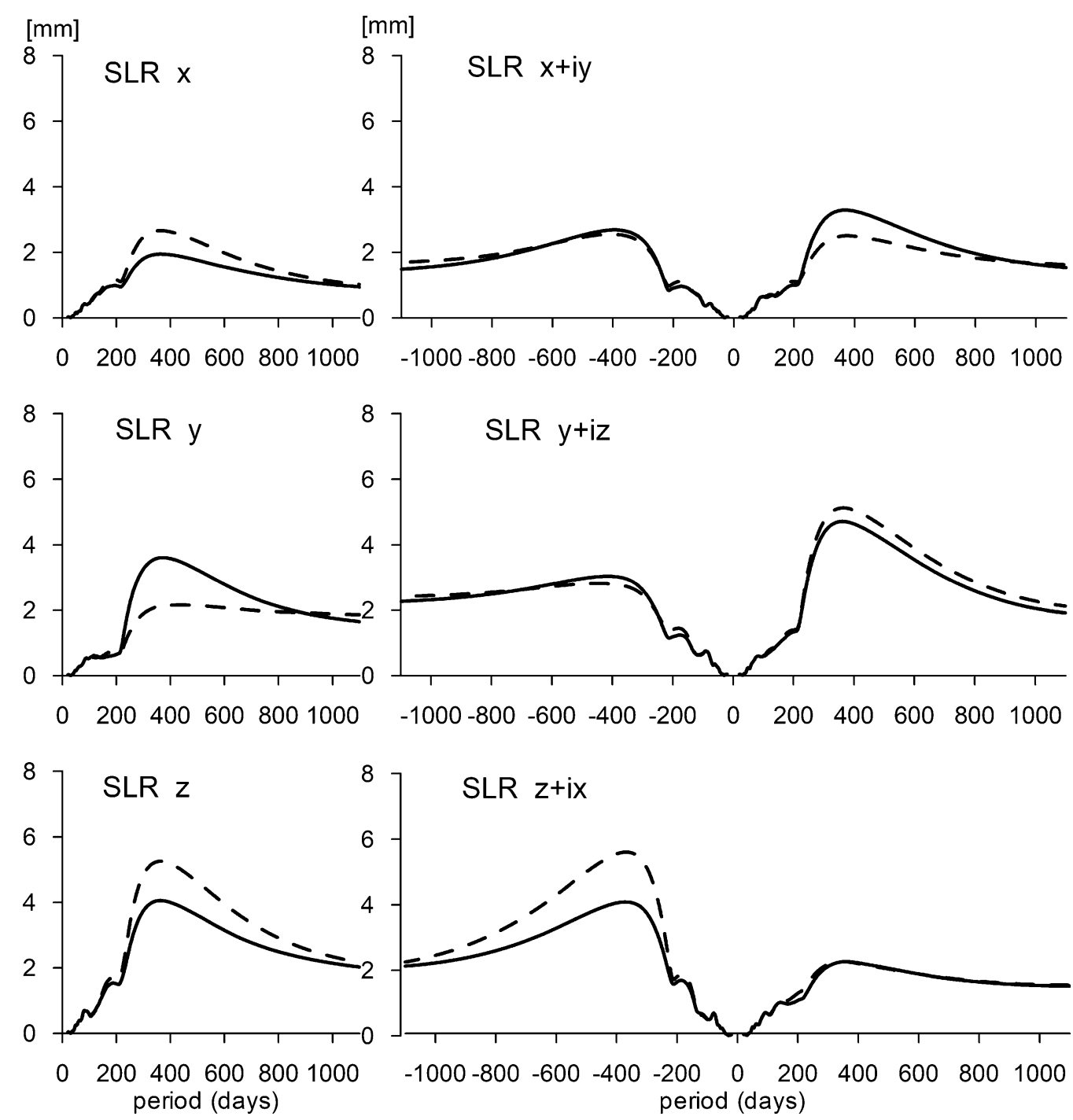

Figure 10

The FTBPF + HT amplitude spectra (left column) of real-valued and the FTBPF amplitude spectra (right column) of complex-valued $\mathrm{X}+\mathrm{iY}, \mathrm{Y}+\mathrm{iZ}$ and $\mathrm{Z}+\mathrm{iX}$ SLR geocenter coordinates (dashed lines) and SLR geocenter residuals after subtracting the weighted mean geocenter model (solid lines)

techniques. We speculate that a similar level of peaks in the prograde and retrograde FTBPF amplitude spectra can be caused by a random polarization character of the annual oscillation. It can be clearly seen that the subtraction of the weighted mean model from DORIS geocenter coordinates causes an increase of the amplitude of the annual oscillation in the $\mathrm{X}$ component (Fig. 7) because the phase of the annual oscillation in the DORIS $X$ coordinate is almost opposite from 2005 to 2013 to the phases of the annual oscillation in other techniques series (Fig. 3). The increase of the amplitude spectrum for the $\mathrm{Z}$ component is caused by the occurrence of the trend in these data.

In the case of the X coordinate for SLR and GNSS techniques, the subtraction of the weighted mean model causes a slight decrease in the amplitude of the annual oscillation (Figs. 8, 10). The subtraction of the weighted mean model from the geocenter coordinates increases the peaks in the FTBPF + HT spectra 
corresponding to the annual oscillation in the $\mathrm{Y}$ and $\mathrm{Z}$ coordinates of the GNSS series and in the Y coordinate of the SLR series as well as in the retrograde FTBPF amplitude spectra of GNSS technique in all planes (Figs. 8, 10).

Because of the systematic errors of the GNSS and SLR techniques in the annual frequency band the amplitudes of annual oscillations in their geocenter time series are different than in the weighted mean model of geocenter coordinates. Consequently, there may occur annual oscillations in both the SLR and GNSS residuals. The differences between the amplitude spectra in the SLR and GNSS geocenter coordinates in the annual oscillation frequency band may result from the fact that the GNSS geocenter series comprises draconitic year oscillations (Meindl et al. 2013) which cannot be resolved from the tropical year in the time series shorter than about 20 years (Thaller et al. 2014). The draconitic GPS period of 351.5 days or the draconitic GLONASS period of 353.2 days cannot be separated from each other nor from the tropical year oscillation by the methods presented in this paper due to too short data span.

The FTBPF + HT and FTBPF amplitude spectra of the DORIS and GNSS geocenter time series and their residuals show peaks in the sub-seasonal frequency band, especially in the $\mathrm{Z}$ coordinate. In the case of GNSS, it may correspond to the 3rd harmonic ( $\sim 117$ days), 5th harmonic ( $\sim 70$ days) and 7 th harmonic ( $\sim 50$ days) of the GNSS draconitic year which has also been noticed by Rebischung et al. (2016a, b). The harmonics of the draconitic year are typically caused by GNSS orbit modeling issues and correlations between empirical orbit parameters and the geocenter coordinates (Rebischung et al. 2013). In DORIS data, the peaks correspond to the frequency of 118 days which may result from systematic errors caused by the solar radiation pressure acting on DORIS satellites (Gobinddass et al. 2009).

\section{Conclusions}

The amplitudes of the annual and semi-annual oscillations computed by the FTBPF + HT are variable in each coordinate of the geocenter time series for all the observation techniques and the semi- annual oscillation amplitude is smaller than the annual one. The mean amplitude of the annual oscillation in the X component is at the level of $2 \mathrm{~mm}$ and agrees well for all considered techniques. In case of the $\mathrm{Y}$ component the mean annual oscillation is equal to $2.4 \mathrm{~mm}$ for SLR, $3.6 \mathrm{~mm}$ for GNSS and in the $\mathrm{Z}$ component the values vary from $2.8 \mathrm{~mm}$ for GRACE + OBP to $5.6 \mathrm{~mm}$ for GNSS. Generally, the phases of the annual oscillation agree in each technique except for the DORIS series for which in the $\mathrm{X}$ and $\mathrm{Z}$ coordinates they are different from the other techniques. The phase variations of the semi-annual oscillation assume values from the entire phase interval $\left[0,360^{\circ}\right]$ and therefore the mean phase values cannot be unambiguously designated.

The common geocenter signals detected in pairs of geocenter coordinates determined by the four independent space-geodetic techniques: DORIS, GNSS, GRACE + OBP, and SLR, using the WBSF, enable the computation of common geocenter motion models in which the annual oscillation is dominant. There is a good phase agreement between weighted mean, average, and median models computed using the WBSF outputs for each coordinate. Due to the computation of the WBSF outputs with similar phases of oscillations the dominant annual present in these models suggests that the proposed weighted mean geocenter motion model may represent the real geocenter motion caused by mass redistribution in the atmosphere, ocean and continental hydrology. The amplitudes of the annual signal agree well for the $\mathrm{X}$ coordinate, however, in the Y coordinate component the amplitudes of the average and median models are a little smaller than that of the weighted mean model. In the XY equatorial plane, the annual oscillation in the computed geocenter motion models is mostly retrograde and in the ZX plane, prograde and retrograde amplitudes are of the same level which means that its polarization is rather chaotic. The signals in two techniques which do not have similar phases should be present in the differences between geocenter coordinates and their weighted mean models. The amplitude spectra of such differences show peaks in the annual frequency band which may correspond to the systematic errors of techniques caused by mismodeling of satellite orbits e.g. draconitic signal of 352 days in the GNSS series. 
The amplitude spectra of the DORIS and GNSS geocenter time series and their residuals show also peaks in the sub-seasonal frequency band, which may correspond to systematic errors caused by mis-modeling of the solar radiation pressure acting on the satellites orbits: $\sim 118$ day oscillation in all components of the DORIS technique and $\sim 117$ and $\sim 70$ days oscillations in the $\mathrm{Z}$ component of the GNSS technique.

Confirmation of the retrograde geocenter motion in the equatorial plane and its geophysical interpretation should also be the motivation for further studies in the future.

The WBSF technique can be recommended to any geodetic time series e.g. Earth orientation parameters or variations of station coordinates determined by different space geodetic techniques of different precision of the measurements to separate geophysical signal which can be represented by the weighted mean WBSF model from systematic errors in each technique.

\section{Acknowledgements}

This project was financed from the funds of the National Science Centre (Poland) allocated on the basis of the decision number DEC-2013/09/N/ST10/ 00664. The research work of Wiesław Kosek was supported by the Polish Ministry of Science and Education, project UMO-2012/05/B/ST10/02132 under the leadership of Prof. A. Brzeziński. The computation algorithms of the WBSF, as well as the FTBPF and the FTBPF + HT amplitude spectra, were elaborated by Wiesław Kosek and Waldemar Popiński.

Open Access This article is distributed under the terms of the Creative Commons Attribution 4.0 International License (http:// creativecommons.org/licenses/by/4.0/), which permits unrestricted use, distribution, and reproduction in any medium, provided you give appropriate credit to the original author(s) and the source, provide a link to the Creative Commons license, and indicate if changes were made.

Publisher's Note Springer Nature remains neutral with regard to jurisdictional claims in published maps and institutional affiliations.

\section{REFERENCES}

Abbondanza, C., Chin, T. M., Gross, R. S., Heflin, M. B., Parker, J., Soja, B. S., et al. (2017). JTRF2014, the JPL Kalman filter, and smoother realization of the International Terrestrial Reference System. Journal of Geophysical Research: Solid Earth, 122, 8474-8510. https://doi.org/10.1002/2017JB014360.

Altamimi, Z., Rebischung, P., Métivier, L., \& Collilieux, X. (2016). ITRF2014: A new release of the international terrestrial reference frame modeling nonlinear station motions. Journal of Geophysical Research: Solid Earth, 121, 6109-6131. https://doi. org/10.1002/2016JB013098.

Arnold, D., Meindl, M., Beutler, G., Dach, R., Schaer, S., Lutz, S., et al. (2015). CODE's new solar radiation pressure model for GNSS orbit determination. Journal of Geodesy, 89(8), 775-791. https://doi.org/10.1007/s00190-015-0814-4.

Bettadpur, S. (2018). UTCSR Level-2 Processing Standards Document (For Level-2 Product Release 0006) (Rev. 5.0, April 18, 2018). GRACE Publication 327-742. 2018. Available online: ftp://isdcftp.gfz-potsdam.de/grace/DOCUMENTS/Level-2/ (for Level-2 Product Release 0006).

Blewitt, G., \& Clarke, P. (2003). Inversion of Earth's changing shape to weigh sea level in static equilibrium with surface mass redistribution. Journal of Geophysical Research: Solid Earth, 108, B6.

Blewitt, G., Lavallée, D., Clarke, P., \& Nurutdinov, K. (2001). A new global mode of Earth deformation: seasonal cycle detected. Science, 294(5550), 2342-2345. https://doi.org/10.1126/science. 1065328.

Bouille, F., Cazenave, A., Lemoine, J. M., \& Cretaux, J. F. (2000). Geocenter motion from the DORIS space system and laser data on Lageos satellites: Comparison with surface loading data. Geophysical Journal International, 143(1), 71-82.

Chambers, P. D., Wahr, J., \& Nerem, R. S. (2004). Preliminary observations of global ocean mass variations with GRACE. Geophysical Research Letters, 31, L13310. https://doi.org/10. 1029/2004GL020461.

Chen, J. L., Rodell, M., Wilson, C. R., \& Famiglietti, J. S. (2005). Low degree spherical harmonic influences on Gravity Recovery and Climate Experiment (GRACE) water storage estimates. Geophysical Research Letters, 32, L14405. https://doi.org/10. 1029/2005GL022964.

Cheng, M.K., Ries, J.C., Tapley, B.D. (2013). Geocenter variations from analysis of SLR Data. Reference Frames for Applications in Geosciences-International Association of Geodesy Symposia 138, 19-25. https://doi.org/10.1007/978-3-642-32998-2_4

Cheng, M. K., \& Tapley, B. D. (2004). Variations in the Earth's oblateness during the past 28 years. Journal of Geophysical Research: Solid Earth, 109, B9. https://doi.org/10.1029/ 2004jb003028.

Collilieux, X., Altamimi, Z., Ray, J., van Dam, T., \& Wu, X. (2009). Effect of the satellite laser ranging network distribution on geocenter motion estimation. Journal of Geophysical Research, 114, B04402. https://doi.org/10.1029/2008JB005727.

Collilieux, X., van Dam, T., Ray, J., Coulot, D., Métivier, L., \& Altamimi, Z. (2012). Strategies to mitigate aliasing of loading signals while estimating GPS frame parameters. Journal of Geodesy, 86(1), 1-14.

Cooper, G. R. J. (2009). Wavelet based semblance filtering. Computers \& Geosciences, 35, 1988-1991. 
Crétaux, J.-F., Soudarin, L., Davidson, F. J. M., Gennero, M.-C., Berge-Nguyen, M., \& Cazenave, A. (2002). Seasonal and interannual geocenter motion from SLR and DORIS measurements: Comparison with surface loading data. Journal of Geophysical Research, 107(B12), 2374. https://doi.org/10.1029/ 2002JB001820.

Dach, R., Lutz, S., Walser, P., Fridez P. (Eds) (2015). Bernese GNSS Software Version 5.2. User manual, Astronomical Institute, University of Bern, Bern Open Publishing. https://doi.org/ 10.7892/boris.72297; ISBN: 978-3-906813-05-9.

Dobslaw, H., Bergmann-Wolf, I., Dill, R., Poropat, L., \& Flechtner, F. (2017). Product description document for AOD1B Release 06 (Rev. 6.1). GRACE Document 327-750, Technical report. GeoForschungsZentrum Potsdam, Potsdam, Germany.

Dong, D., Dickey, J. O., Chao, Y., \& Cheng, M. K. (1997). Geocenter variations caused by atmosphere, ocean and surface ground water. Geophysical Research Letters, 24(15), 1867-1870.

Dong, D., Qu, W., Fang, P., \& Peng, D. (2014). Non-linearity of geocenter motion and its impact on the origin of the terrestrial reference frame. Geophysical Journal International, 198(2), 1071-1080. https://doi.org/10.1093/gji/ggu187.

Dong, D., Yunck, T., \& Heflin, M. (2003). Origin of the international terrestrial reference frame. Journal of Geophysical Research, 108, B4. https://doi.org/10.1029/2002jb002035.

Feissel-Vernier, M., Le Bail, K., Berio, P., Coulot, D., Ramillien, G., \& Valette, J.-J. (2006). Geocenter motion measured with DORIS and SLR, and predicted by geophysical models. Journal of Geodesy, 80(8-11), 637-648. https://doi.org/10.1007/s00190006-0079-z.

Ferland, R., \& Piraszewski, M. (2009). The IGS-combined station coordinates, earth rotation parameters and apparent geocenter. Journal of Geodesy, 83(3-4), 385-392.

Frazier, M., \& Torres, R. (1994). The sampling theorem, $\varphi$-transform, and Shannon wavelets for $\mathrm{R}, \mathrm{Z}, \mathrm{T}$ and $\mathrm{Z}_{\mathrm{N}}$. In $\mathrm{J}$. J. Benedetto \& M. W. Frazier (Eds.), Wavelets-mathematics and applications (pp. 221-245). Boca Raton: CRC Press.

Gasquet, C., \& Witomski, P. (1999). Fourier analysis and applications-filtering, numerical computation, wavelets. New York: Springer. https://doi.org/10.1007/978-1-4612-1598-1.

Glaser, S., Fritsche, M., Sośnica, K., Rodríguez-Solano, C. J., Wang, K., Dach, R., et al. (2015a). A consistent combination of GNSS and SLR with minimum constraints. Journal of Geodesy. https://doi.org/10.1007/s00190-015-0842-0.

Glaser, S., Fritsche, M., Sośnica, K., Rodríguez-Solano, C. J., Wang, K., Dach, R., et al. (2015b). A consistent combination of GNSS and SLR with minimum constraints. Journal of Geodesy, 89(12), 1165-1180.

Gobinddass, M. L., Willis, P., De Viron, O., Sibthorpe, A., Zelensky, N. P., Ries, J. C., et al. (2009). Improving DORIS geocenter time series using an empirical rescaling of solar radiation pressure models. Advances in Space Research, 44(11), 1279-1287.

Guo, J., Han, Y., \& Hwang, C. (2008). Analysis on motion of Earth's center of mass observed with CHAMP mission. Science in China Series G: Physics, Mechanics and Astronomy, 51(10), 1597-1606. https://doi.org/10.1007/s11433-008-0152-0.

Haines, B. J., Bar-Sever, Y. E., Bertiger, W. I., Desai, S. D., Harvey, N., Sibois, A. E., et al. (2015). Realizing a terrestrial reference frame using the Global Positioning System. Journal of Geophysical Research: Solid Earth, 120(8), 5911-5939.
Kang, Z. G., Tapley, B., Chen, J. L., Ries, J., \& Bettadpur, S. (2009). Geocenter variations derived from GPS tracking of the GRACE satellites. Journal of Geodesy, 83(10), 895-901. https:// doi.org/10.1007/s00190-009-0307-4.

Klemann, V., \& Martinec, Z. (2009). Contribution of glacial-isostatic adjustment to the geocenter motion. Tectonophysics, 511(3-4), 99-108. https://doi.org/10.1016/j.tecto.2009.08.031.

Kosek, W. (1995). Time variable band pass filter spectra of real and complex-valued polar motion series. Artificial Satellites, Planetary Geodesy, 30(1), 27-43.

Kosek, W. (2002). Autocovariance prediction of complex-valued polar motion time series. Advances of Space Research, 30, 375-380.

Kosek, W., Niedzielski, T., Popiński, W., Zbylut-Górska, M., Wnęk, A. (2015). Variable seasonal and subseasonal oscillations in sea level anomaly data and their impact on prediction accuracy. Chapter, Part of the series International Association of Geodesy Symposia, vol. 142, Proceedings of the VIII Hotine Marussi Symposium, 17-21 June 2013, Rome, Italy, Sneeuw N., Novák P., Crespi M., Sansò F. (eds), pp 1-4. https://doi.org/10. 1007/1345_2015_74

Kosek, W., Wnęk, A., Zbylut, M., \& Popiński, W. (2014). Wavelet analysis of the Earth center of mass time series determined by satellite techniques. Journal of Geodynamics, 80, 58-65. https:// doi.org/10.1016/j.jog.2014.02.005.

Kuang, D., Bar-Sever, Y., \& Haines, B. (2015). Analysis of orbital configurations for geocenter determination with GPS and lowEarth orbiters. Journal of Geodesy, 89(5), 471-481. https://doi. org/10.1007/s00190-015-0792-6.

Lavallée, D. A., van Dam, T., Blewitt, G., \& Clarke, P. J. (2006). Geocenter motions from GPS: a unified observation model. Journal of Geophysical Research: Solid Earth, 111, B5. https:// doi.org/10.1029/2005jb003784.

Li, Y., Song, S., Zhu, W., Zhao J. (2012). Seasonal variations analysis of the origin and scale of international terrestrial reference frame. Chapter, China Satellite Navigation Conference (CSNC) 2012 Proceedings, vol. 160 of the series lecture notes in electrical engineering, pp. 253-268. https://doi.org/10.1007/9783-642-29175-3_23

Meindl, M., Beutler, G., Thaller, D., Dach, R., \& Jäggi, A. (2013). Geocenter coordinates estimated from GNSS data as viewed by perturbation theory. Advances in Space Research, 51(7), 1047-1064. https://doi.org/10.1016/j.asr.2012.10.026.

Meindl, M., Beutler, G., Thaller, D., Dach, R., Schaer, S., \& Jäggi, A. (2015). A comment on the article "A collinearity diagnosis of the GNSS geocenter determination" by P. Rebischung, Z. Altamimi, and T. Springer. Journal of Geodesy, 89(2), 189-194. https://doi.org/10.1007/s00190-014-0765-1.

Pearlman, M., Arnold, D., Davis, M., Barlier, F., Biancale, R., Vasiliev, V., et al. (2019). Laser geodetic satellites: a high-accuracy scientific tool. Journal of Geodesy. https://doi.org/10. 1007/s00190-019-01228-y.

Petit, G., \& Luzum, B. (Eds.). (2010). IERS Conventions. IERS Technical Note No. 36, Frankfurt am Main: Verlag des Bundesamts für Kartographie und Geodäsie. ISBN 3-89888-989-6.

Popiński, W. (2008). Insight into the fourier transform band pass filtering technique. Artificialm Satellites, 43(4), 129-141.

Rebischung, P., Altamimi, Z., Ray, J., \& Garayt, B. (2016a). The IGS contribution to ITRF2014. Journal of Geodesy, 2016, 1-20. https://doi.org/10.1007/s00190-016-0897-6. 
Rebischung, P., Altamimi, Z., Ray, J., \& Garayt, B. (2016b). The IGS contribution to ITRF2014. Journal of Geodesy, 90(7), 611-630.

Rebischung, P., Altamimi, Z., \& Springer, T. (2013). A collinearity diagnosis of the GNSS geocenter determination. Journal of Geodesy, 88(1), 65-85. https://doi.org/10.1007/s00190-0130669-5.

Rietbroek, R., Fritsche, M., Brunnabend, S., Daras, I., Kusche, J., Schröter, J., et al. (2012). Global surface mass from a new combination of GRACE, modelled OBP and reprocessed GPS data. Journal of Geodynamics, 59-60, 64-71. https://doi.org/10. 1016/j.jog.2011.02.003.

Sośnica, K., Thaller, D., Dach, R., Jäggi, A., \& Beutler, G. (2013). Impact of loading displacements on SLR-derived parameters and on the consistency between GNSS and SLR results. Journal of Geodesy, 87(8), 751-769. https://doi.org/10.1007/s00190-0130644-1.

Soudarin, L., \& Ferrage, P. (Eds.). (2017). International DORIS service activity report 2017. Available online: https://ids-doris. org/documents/report/IDS_Report_2017.pdf.

Sun, Y., Ditmar, P., \& Riva, R. (2017). Statistically optimal estimation of degree- 1 and $\mathrm{C} 20$ coefficients based on GRACE data and an ocean bottom pressure model. Geophysical Journal International, 210(3), 1305-1322.

Sun, Y., Riva, R., \& Ditmar, P. (2016). Optimizing estimates of annual variations and trends in geocenter motion and $\mathrm{J} 2$ from a combination of GRACE data and geophysical models. Journal of Geophysical Research: Solid Earth, 121(11), 8352-8370.

Swenson, S., Chambers, D., \& Wahr, J. (2008). Estimating geocenter variations from a combination of GRACE and ocean model output. Journal of Geophysical Research: Solid Earth, 113, B8. https://doi.org/10.1029/2007jb005338.

Tapley, B., Bettadpur, S., Watkins, M., \& Reigber, M. (2004). The gravity recovery and climate experiment: mission overview and early results. Geophysical Research Letters, 31(9), L09607. https://doi.org/10.1029/2004GL019920.
Thaller, D., Sośnica, K., Dach, R., Jäggi, A., Beutler, G., Mareyen, M., et al. (2014). Geocenter coordinates from GNSS and combined GNSS-SLR solutions using satellite co-locations. Earth on the Edge: Science for a Sustainable Planet, International Association of Geodesy Symposia, 139(2014), 129-134. https://doi. org/10.1007/978-3-642-37222-3_16.

Willis, P., Fagard, H., Ferrage, P., Lemoine, F.G., Noll, C.E., Noomen, R., Otten, M., Ries, J.C., Rothacher, M., Soudarin, L., Tavernier, G., Valette, J.J. (2010). The international DORIS service (IDS): Toward maturity, in DORIS: Scientific Applications in Geodesy and Geodynamics, P. Willis (Ed.), Advances in Space Research, 45(12), 1408-1420, https://doi.org/10.1016/j. asr.2009.11.018

Wu, X., Abbondanza, C., Altamimi, Z., Chin, T. M., Collilieux, X., Gross, R. S., et al. (2015). KALREF-A Kalman filter and time series approach to the International Terrestrial Reference Frame realization. Journal of Geophysical Research: Solid Earth, 120, 3775-3802. https://doi.org/10.1002/2014JB011622.

Wu, X., Argus, D. F., Heflin, M. B., Ivins, E. R., \& Webb, F. H. (2002). Site distribution and aliasing effects in the inversion for load coefficients and geocenter motion from GPS data. Geophysical Research Letters, 29(24), 2210. https://doi.org/10.1029/ 2002 GL016324.

Wu, X., Collilieux, X., Altamimi, Z., Vermeersen, B. L. A., Gross, R. S., \& Fukumori, I. (2011). Accuracy of the international terrestrial reference frame origin and earth expansion. Geophysical Research Letters, 38, 13. https://doi.org/10.1029/2011g1047450.

Wu, X., Kusche, J., \& Landerer, W. F. (2017). A New unified approach to determine geocenter motion using space geodetic and GRACE gravity data. Geophysical Journal International. https://doi.org/10.1093/gji/ggx086.

Wu, X., Ray, J., \& van Dam, T. (2012). Geocenter motion and its geodetic and geophysical implications. Journal of Geodynamics, 58, 44-61. https://doi.org/10.1016/j.jog.2012.01.007. (ISSN 0264-3707). 